\title{
Oxytocin promotes epicardial cell activation and heart regeneration after cardiac injury
}

Aaron H. Wasserman ${ }^{1,2}$, Yonatan R. Lewis-Israeli ${ }^{1,2}$, Amanda R. Huang ${ }^{1,2}$, McKenna D. Dooley $^{1,2}$, Allison L. Mitchell ${ }^{1,2}$, Manigandan Venkatesan ${ }^{1,2}$ and Aitor Aguirre ${ }^{1,2}$

${ }^{1}$ Division of Developmental and Stem Cell Biology, Institute for Quantitative Health Science and Engineering (IQ), Michigan State University, East Lansing, MI, USA

${ }^{2}$ Department of Biomedical Engineering, College of Engineering, Michigan State University, East Lansing, MI, USA

Corresponding author: aaguirre@msu.edu 


\section{ABSTRACT}

Cardiovascular disease (CVD) is one of the leading causes of mortality worldwide, and frequently leads to massive heart injury and the loss of billions of cardiac muscle cells and associated vasculature. Critical work in the last two decades demonstrated that these lost cells can be partially regenerated by the epicardium, the outermost mesothelial layer of the heart, in a process that highly recapitulates its role in heart development. Upon cardiac injury, mature epicardial cells activate and undergo an epithelial-mesenchymal transition (EMT) to form epicardial-derived progenitor cells (EpiPCs), multipotent progenitors that can differentiate into several important cardiac lineages, including cardiomyocytes and vascular cells. In mammals, this process alone is insufficient for significant regeneration, but it may be possible to prime it by administering specific reprogramming factors, leading to enhanced EpiPC function. Here, we show that oxytocin (OXT), a hypothalamic neuroendocrine peptide, induces epicardial cell proliferation, EMT, and migration in a mature-like model of human induced pluripotent stem cell (hiPSC)-derived epicardial cells. In addition, we demonstrate that OXT is released from the brain into the bloodstream after cardiac cryoinjury in zebrafish, eliciting significant epicardial activation and promoting heart regeneration. Oxytocin signaling is also critical for proper epicardium and myocardium development in zebrafish embryos. The above processes are significantly impaired when OXT signaling is inhibited chemically and genetically through RNA interference. Mechanistically, RNA sequencing analyses suggest that the transforming growth factor beta (TGF- $\beta$ ) pathway is the primary mediator of OXT-induced epicardial activation. Our research reveals for the first time a primarily brain-controlled mechanism that induces cellular reprogramming and regeneration of the injured heart, a finding that could yield significant translational advances for the treatment of CVD.

KEYWORDS: Heart Regeneration, Epicardium, Oxytocin, Zebrafish, Cell Reprogramming, Stem Cells, Induced Pluripotent Stem Cells, Epicardium-Derived Stem Cells 


\section{INTRODUCTION}

Cardiovascular disease (CVD) is the leading cause of mortality in the United States and the rest of the developed world (Virani et al., 2020). These diseases often lead to severe cardiac events, such as a myocardial infarction (MI), which can result in the death of more than $25 \%$ of cardiac myocytes, the chief contractile cells of the adult heart. If left untreated, this condition can lead to heart failure (Laflamme \& Murry, 2011). Cardiomyocytes (CMs) are terminally differentiated cells, and as such, their innate regenerative capacity is extremely limited and insufficient to restore function to lost myocardium. Instead, the injured heart typically resorts to wound repair, replacing dead cells with fibrotic scar tissue (Bergmann et al., 2009; Fan et al., 2012). Therefore, the ability to induce heart regeneration, which results in growth and proliferation of endogenous diseased cells (and subsequent restoration of cardiac function), would be paramount in the discovery of new therapeutic strategies for the successful treatment of $\mathrm{Ml}$ and other types of CVD (Laflamme \& Murry, 2011; Uygur \& Lee, 2016).

The epicardium is the outermost mesothelial layer of the heart that serves a structural and protective role in healthy individuals (Riley, 2012). While its activity level decreases as development proceeds, in adults, it becomes activated upon injury and recapitulates its embryonic phenotype (Peralta et al., 2014; Simões \& Riley, 2018). Upon activation, a subset of epicardial cells migrate into the subepicardial space and undergo epithelial-mesenchymal transition (EMT) to form epicardium-derived progenitor cells (EpiPCs). These EpiPCs are multipotent cardiac progenitors (the only widely accepted source) that contribute to multiple different cell lineages in the heart and can aid in the healing and repair process (Quijada et al., 2020; Rao \& Spees, 2017; Smits et al., 2018). While there is evidence to suggest that EpiPCs can differentiate into cardiomyocytes during development (Cai et al., 2008; Zhou et al., 2008), whether this process occurs in adult EpiPCs is a matter of ongoing debate. Adult epicardial cells also secrete paracrine factors to modulate heart repair and regeneration post-MI (Wei et al., 2015; Zhou et al., 2011). Therefore, the activated epicardium might be the key to unlocking cardiac regeneration pathways in humans.

The brain plays a central role in several energetically demanding processes that serve to maintain body homeostasis (Dampney, 2016; Guyenet \& Bayliss, 2015; Morrison, 2016; Roh et al., 2016). In addition, the brain-heart signaling axis regulates heart rate, blood pressure, and systolic and diastolic function (Dampney, 2016; Palma \& Benarroch, 2014). The neural endocrine structures, such as the hypothalamus and pituitary, play a particularly important role in maintaining normal cardiovascular function through the release of various hormones (Gordan et al., 2015; Rhee \& Pearce, 2011). Therefore, it stands to reason that a process as biologically and energetically demanding as heart regeneration does not happen cellautonomously and might be under central hormonal control. In fact, there is evidence that several hormones such as estrogen (Xu et al., 2020), thyroid hormones (Hirose et al., 2019), and cortisol (Sallin \& Jaźwińska, 2016) affect cardiac regeneration. In addition, a recent study indicates that damage to the hypothalamus inhibits endogenous regenerative processes in vertebrates (Zhang et al., 2018). These findings, combined with the fact that injury-induced epicardial activation can be primed by specific signaling factors (Smart et al., 2011), led us to hypothesize that after cardiac injury, one or more neuroendocrine hormones are released from the hypothalamus into the bloodstream to facilitate heart regeneration. Proper identification and characterization of these hormones will be critical in advancing the translational potential of cardiac regeneration research. 
Here, we present evidence that oxytocin (OXT), a nine amino acid neuropeptide produced by the hypothalamus and released by the posterior pituitary, may be the missing link in achieving sufficient EpiPC proliferation, activation, and migration to fully regenerate the lost myocardium in the injured heart. We utilize both in vitro and in vivo injury models to identify and describe the direct involvement of OXT in these processes. First, we differentiate and characterize a mature-like model of human iPSC-derived epicardial cells (hEpiCs) and show that the peptide induces proliferation and activation in these cells by acting through its receptor (OXTR). We then show that oxytocin is released from the brain after cardiac cryoinjury in zebrafish, a process that is critical for heart regeneration and epicardial activation. In addition, inhibition of OXT signaling delays regeneration of adult hearts and epicardium formation in zebrafish embryos. RNA sequencing (RNA-seq) analyses suggest that the TGF- $\beta$ pathway is the primary mediator of OXT-induced epicardial activation. The findings of this study reveal a novel role for the brain-heart signaling axis in regulating cardiac regeneration and elucidate the critical importance of the epicardium in this process.

\section{MATERIALS AND METHODS}

Stem cell culture. Human iPSCs (hiPSC-L1) were cultured on 6 well plates coated with growth factor-reduced Matrigel (Corning) in an incubator at $37^{\circ} \mathrm{C}, 5 \% \mathrm{CO}_{2}$. Stem cells were maintained in Essential 8 Flex medium (E8 Flex; Thermo Fisher Scientific) containing 1\% penicillin/streptomycin until $\sim 70 \%$ confluency was reached, at which point cells were split into new wells using ReLeSR passaging reagent (Stem Cell Technologies) with $2 \mu \mathrm{M}$ ROCK inhibitor (Thiazovivin/TZV; Selleck Chemicals) added to prevent cells from undergoing apoptosis while in suspension. All hiPSC lines were periodically validated for pluripotency and genomic stability.

hiPSC differentiation to hEpiCs. hiPSCs were differentiated to epicardial monolayers using a modified version of a previously described protocol (Bao et al., 2016, 2017). Cells were dissociated using Accutase (Innovative Cell Technologies), re-plated on Matrigel coated plates, and incubated overnight in E8 Flex $+2 \mu \mathrm{M}$ TZV. Once hiPSC monolayers reached $\sim 90 \%$ confluency, epicardial differentiation was started via the addition of $12 \mu \mathrm{M} \mathrm{CHIR99021}$ (Selleck Chemicals) in RPMI/B-27 minus insulin (Gibco; Day 0) for 24 hours. On Day 3, cells were exposed to $2 \mu \mathrm{M}$ Wnt-C59 (Selleck Chemicals) in RPMI/B-27 minus insulin for 48 hours. On Day 6, cardiac progenitors were then re-plated in RPMI/B-27 (Gibco) with $100 \mu \mathrm{g} / \mathrm{mL}$ Vitamin C (Vit. C) using Accutase and TZV. Cells were directed down the epicardial lineage by the consecutive addition of $9 \mu \mathrm{M}$ CHIR99021 on Days 7 and 8 and maintained in RPMI/B$27 /$ Vit. C until passage. On day 12, hEpiCs were again re-plated using Accutase and TZV and $2 \mu \mathrm{M}$ SB431542 (TGF- $\beta$ receptor inhibitor; Selleck Chemicals) was added to the media to prevent cells from spontaneously undergoing EMT. Epicardial cells were cultured long-term in RPMI/B-27/Vit. C $+2 \mu \mathrm{M}$ SB431542 and split when full confluency was reached. In order to ensure that hEpiCs were as mature as possible for experiments, Vitamin C and SB431542 were entirely removed from the culture system 5 days prior to sample collection. OXT and other compounds were then added directly to the media 2 days later, for a total exposure time of 3 days, unless otherwise indicated. Automated cell counting was conducted using the Cytation 3 and Cytation 5 Cell Imaging Multi-Mode Readers (Biotek).

hiPSC lentiviral transduction. Bacteria carrying the plasmid for shRNA-mediated knockdown of OXTR and a scrambled plasmid (both designed with VectorBuilder) were grown on LB agar plates and isolated colonies were expanded in LB broth, both containing ampicillin. The 
plasmids carried an ampicillin resistance cassette, allowing for their growth. Plasmid DNA was isolated from the bacteria by midiprep (Zymo Research), and purified DNA was transfected into $40 \%$ confluent HEK293T cells using Lipofectamine (Invitrogen). Lentiviral packaging plasmids (pVSVg, psPAX2) were also transfected at this time, thereby allowing the generation of a functional lentivirus containing the shRNA molecules of interest. Viral supernatant was collected and concentrated after 48 hours and transduced directly into hiPSCs at low to midconfluency along with $8 \mu \mathrm{g} / \mathrm{mL}$ polybrene (Fisher Scientific). Because all plasmids contained puromycin-resistance cassettes, the stem cell media was changed the next day to E8 Flex containing $0.5 \mu \mathrm{g} / \mathrm{mL}$ puromycin (Thermo Fisher Scientific) to facilitate colony selection. hiPSCs were maintained in puromycin for 3-5 days and surviving monoclonal colonies were replated and expanded to generate two new hiPSC lines. The extent of OXTR knockdown was then quantified by qRT-PCR in hEpiCs.

Zebrafish cardiac cryoinjury. Adult zebrafish hearts were subjected to cardiac cryoinjury as described previously (González-Rosa et al., 2011; González-Rosa \& Mercader, 2012). Briefly, fish were anesthetized with $0.65 \mathrm{mM}$ tricaine (MS-222) and placed on a wet sponge ventral side up under a stereomicroscope (Leica). The chest cavity was opened by cutting through the pericardium until the point where the beating heart was clearly visible. In order to induce cryoinjury, a liquid nitrogen cooled metal probe was gently placed on the apex of the ventricle for $\sim 45$ seconds. Sham operations were carried out by simply opening the chest cavity without injury. Fish were closely monitored for the first 2 hours after surgery and twice daily thereafter until ready for organ collection. Pharmacological inhibition of oxytocin signaling was achieved by injecting 2-3 $\mu \mathrm{L}$ of water or $1 \mu \mathrm{M} \mathrm{L-368,899}$ (a non-peptide OXTR antagonist) directly into the intrathoracic cavity, as described previously (Bise \& Jaźwińska, 2019). Initial injections were administered 24 hours after cryoinjury with repeat injections every other day throughout the experiment. All zebrafish were maintained in a dedicated facility between $27-28^{\circ} \mathrm{C}$ on a 14-hour light/10-hour dark cycle. Equal numbers of male and female fish were used for cryoinjuries.

Zebrafish embryo experiments. One female and one male adult fish were placed in specialized breeding tanks with a partition between them the night before experiments. The partition was removed early the next morning and fish were given $\sim 15$ minutes to breed. Fertilized embryos were collected and placed in embryo medium containing $4.96 \mathrm{mM} \mathrm{NaCl}, 0.179 \mathrm{mM} \mathrm{KCl}, 0.329$ $\mathrm{mM} \mathrm{CaCl} 2 \cdot 2 \mathrm{H}_{2} \mathrm{O}$, and $0.401 \mathrm{mM} \mathrm{MgCl} 2 \cdot 6 \mathrm{H}_{2} \mathrm{O}$ dissolved in water. The appropriate concentration of atosiban (competitive OXTR antagonist) was added directly to the medium and embryos were stored in an incubator at $28^{\circ} \mathrm{C}$ for the duration of the experiment. Dead embryos and discarded chorions were removed daily. Developing fish were imaged at 24-, 48, and 72-hours post-fertilization (hpf) with the Leica M165 FC stereomicroscope with a DFC7000 T fluorescence camera so that epicardium and myocardium formation could be observed in real time. Epicardial cell number was counted manually at 48 and $72 \mathrm{hpf}$.

Gene expression analysis. Total RNA was extracted from samples using the RNeasy Mini Kit (Qiagen). Cells were lysed directly in their culture wells and tissues were lysed and homogenized using the Bead Mill 4 Homogenizer (Fisher Scientific). Once extracted, RNA was quantified using a NanoDrop (Mettler Toledo), with a concentration of at least $10 \mathrm{ng} / \mu \mathrm{L}$ being required to proceed with reverse transcription. CDNA was synthesized using the Quantitect Reverse Transcription Kit (Qiagen) and stored at $-20^{\circ} \mathrm{C}$ for further use. Primers for qRT-PCR were designed using the Primer Quest tool (Integrated DNA Technologies) and SYBR Green (Qiagen) was used as the DNA intercalating dye. qRT-PCR plates were run 
using the QuantStudio 5 Real-Time PCR system (Applied Biosystems) with a total reaction volume of $20 \mu \mathrm{L}$. Expression levels of genes of interest were normalized to HPRT1 levels and fold change values were obtained using the $2^{-\Delta \Delta C T}$ method. At least 3-5 independent samples were run for each gene expression assay.

Immunofluorescence. hEpiC samples were first transferred onto Millicell EZ slides (MilliporeSigma), fixed in 4\% paraformaldehyde (PFA) solution, and washed with PBS +1.5 $\mathrm{g} / \mathrm{L}$ glycine. Zebrafish tissue samples were extracted, washed in PBS + $0.5 \mathrm{mM}$ EDTA solution, and fixed in 4\% PFA for one hour. After fixation, organs were washed with PBSglycine and transferred into a PBS $+30 \%$ sucrose solution for at least 48 hours. Samples were then embedded in Optimal Cutting Temperature (OCT) compound (Electron Microscopy Sciences), frozen in a mold, and sectioned at $10 \mu \mathrm{m}$ thickness using the Leica CM3050 S cryostat. Sectioned tissue was stored at $4^{\circ} \mathrm{C}$ for further use. Once ready for staining, antigen retrieval was performed by immersing slides in a sodium citrate buffer $(10 \mathrm{mM}$ sodium citrate, $0.05 \%$ Tween $20, \mathrm{pH} 6.0$ ) for $\sim 45$ minutes at $95-100^{\circ} \mathrm{C}$. All samples (cells and tissues) were blocked and permeabilized with $10 \%$ normal donkey serum, $0.5 \%$ bovine serum albumin (BSA), and $0.5 \%$ Triton X-100 in PBS for 1 hour at room temperature. After washing, primary antibodies were diluted in antibody solution ( $1 \%$ normal donkey serum, $0.5 \%$ BSA, $0.5 \%$ Triton X-100 for tissues, $0.05 \%$ Triton X-100 for cells, all dissolved in PBS), added to the samples, and incubated overnight at $4{ }^{\circ} \mathrm{C}$. The next day, cells and tissues were washed, and secondary antibodies were diluted in antibody solution (with $0.05 \%$ Triton X-100) and added for 2 hours at room temperature. 4',6-diamidino-2-phenylindole (DAPI, Thermo Fisher Scientific) was then added immediately at a concentration of 1:1000 to label DNA. Stained slides were washed 3 times in PBS and No.1 coverslips (VWR) were added using ProLong Gold Antifade Mountant (Thermo Fisher Scientific).

Histology. Masson's trichrome staining was carried out using a kit from IMEB Inc. and following manufacturer's instructions. Briefly, sectioned tissue was immersed in Bouin's solution for $~ 50$ minutes at $56-64^{\circ} \mathrm{C}$ to improve staining quality and washed in tap water until the yellow color was fully removed from the hearts. Slides were then stained with Biebrich's scarlet/acid fuchsin solution for 7 minutes, washed in deionized (DI) $\mathrm{H}_{2} \mathrm{O}$, and immersed in phosphomolybdic/phosphotungstic acid solution for 18 minutes to decolorize fibrous tissue. Slides were then transferred directly to aniline blue solution for 12 minutes to stain collagen fibers, washed in $\mathrm{DI} \mathrm{H}_{2} \mathrm{O}$, and immersed in $1 \%$ acetic acid for 3 minutes to stabilize the staining. After washing, tissue was dehydrated by successive exposure to $95 \%$ ethanol, $100 \%$ ethanol, and xylene and coverslips were added using Eukitt quick-hardening mounting medium (Sigma-Aldrich).

Confocal microscopy and image analysis. All samples were imaged using the Zeiss LSM880 NLO Confocal Microscope system or the Nikon A1 Confocal Laser Microscope. Images were analyzed and prepared for publication using Fiji software. Cell counts were performed manually using the "Wand" tool and automatically using the "Threshold" and "Analyze Particles" tools. Individuals who conducted manual cell counting were blinded to experimental conditions and at least 5 images were analyzed for each group.

RNA sequencing. RNA was extracted from 3 control and $3 \mathrm{hEpiC}$ samples treated with $100 \mathrm{nM}$ OXT as described above. RNA was quantified using a Qubit Fluorometer (Thermo Fisher Scientific) and samples were sent to the MSU Genomics core, where their quality was tested using the Agilent 2100 Bioanalyzer. Samples were sequenced using an Illumina HiSeq 4000. 
For RNA-seq sample processing, a pipeline was created in Galaxy. Briefly, sample run quality was assessed with FASTQC and alignment to hg38 was carried out using HISAT2. Counts were obtained using featureCounts and differential expression analysis was performed with EdgeR. Further downstream bioinformatic analysis was performed using Phantasus 1.11.0 (artyomovlab.wustl.edu/phantasus), ToppGene Suite (http://toppgene.cchmc.org), and Enrichr (https://maayanlab.cloud/Enrichr/).

Statistical analysis. All graphs were made using GraphPad software. Statistical significance was evaluated with a standard unpaired Student's t-test (2-tailed) when comparing two groups or with a 1-way ANOVA with Tukey's or Dunnett's post-test for multiple comparison analysis. Regardless of the test used, a P-value of less than 0.05 was considered statistically significant. All data are presented as mean \pm SD or SEM and represent a minimum of 3 independent experiments with at least 3 technical replicates unless otherwise stated. For RNA-seq analysis, a false discovery rate (FDR) was used to determine statistical significance.

\section{RESULTS}

Establishing human iPSC-derived epicardial progenitor and mature-like epicardial cell cultures. To produce mature-like epicardial cells from human iPSCs (hEpiCs) we started by deriving epicardial progenitor cells using a 3-step Wnt modulation strategy (Bao et al., 2016, 2017) (Figure 1A). After 12 days of culture, hEpiCs display classic epithelial morphology (Figure 1B), express epicardial progenitor markers WT1 and TCF21 (Figure 1C-E), and show increased $W T 1$ and $C D H 1$ (epithelial cadherin) levels as they age (Figure 1F, G), suggesting an increase in epicardial nature. These cells can be routinely maintained and cultured in medium containing Vitamin C and SB431542 (a TGF- $\beta$ receptor inhibitor), allowing them to retain their epithelial morphology for at least 20 passages; however, we found that these compounds prevent $\mathrm{hEpiC}$ maturation. As the epicardium develops and ages, its resident cells transition from a highly proliferative, migratory, and transcriptionally active nature to a more mature, quiescent phenotype. In the mouse heart, key genes involved in epicardial activation are barely detectable by 3 months of age (Smart et al., 2011; Smits et al., 2018; Zhou et al., 2011). We hypothesized that removing Vitamin $C$ and SB431542 from the hEpiC media for a short time would allow the cells to mature without undergoing EMT. Indeed, we found that hEpiC cell counts were 2-fold higher when cultured in media containing these compounds compared to when they were absent (Figure 1H). In addition, removal of SB431542 from the media five days prior to sample collection led to hEpiC transcriptional maturation, as shown by a decrease in progenitor and EMT markers (WT1, TCF21, NT5E) and an increase in mature epithelial marker $\mathrm{CDH1}$ (Figure 1I). Therefore, all experiments with epicardial cells were performed using this final media formulation to ensure that hEpiCs resemble epicardial cells in the adult heart as much as possible.

Oxytocin induces human epicardial cell activation to a progenitor-like state. Because the brain is critical for maintenance of body homeostasis (Dampney, 2016; Guyenet \& Bayliss, 2015; Morrison, 2016; Roh et al., 2016) and damage to the hypothalamus inhibits limb regeneration in vertebrates (Zhang et al., 2018), we hypothesized that the brain neuroendocrine system is also involved in the process of heart regeneration. To identify potential new neuroendocrine hormones involved in epicardial activation and possibly heart regeneration, we screened fifteen endogenous candidates in hEpiCs with two main criteria in mind: 1) Being involved in the physiological response to stress and 2) Being produced and secreted by neuroendocrine tissue (see Supplementary Table 1). We identified 15 
candidates and screened each of them for their proliferative effects by adding them to mature hEpiCs in culture. After 3 days, the percentage of phosphorylated histone $\mathrm{H} 3(\mathrm{H} 3 \mathrm{P})$ positive cells was measured as a putative marker of proliferation. Oxytocin (OXT), a neuroendocrine peptide released by the neurohypophysis, had the strongest proliferative effects ( 3-fold versus control) and was selected for further downstream analysis (Figure 2A). We then exposed hEpiCs to different OXT concentrations in the physiological range and assessed cellular proliferation levels by two alternative methods, Ki67 staining and direct cell number counts. We found that the percentage of hEpiCs double-positive for both WT1 and Ki67 increased by $\sim 50 \%$ three days after addition of OXT (Figure 2B, C). Automated direct cell counting using DAPI staining produced similar results and indicated that the absolute number of epicardial cells doubled with oxytocin exposure (Figure 2D). No multinucleated cells were observed. We compared the effect of OXT to that of thymosin $\beta 4$, a short peptide that has been shown to elicit strong epicardial activation in vitro and epicardial-mediated heart regeneration in vivo in mice (Smart et al., 2007, 2011; Y. L. Wang et al., 2021). $100 \mathrm{nM}$ oxytocin again caused a $\sim 50 \%$ increase in hEpiC proliferation compared to control cells, an effect that was 2-fold greater than that of the most potent thymosin $\beta 4$ dose (Figure 2E). OXT also induced $\mathrm{hEpiC}$ epithelial-to-mesenchymal transition (EMT), as determined by an increase in gene expression for EMT markers WT1, TCF21, and SNAl1 without a corresponding increase in epithelial marker CDH1 (Figure 2F). Taken together, these data suggest that oxytocin induces epicardial cell activation to a progenitor-like state.

Oxytocin exerts its pro-regenerative effects through the oxytocin receptor (OXTR). We sought to gain some mechanistic insight as to how OXT causes its effects in vitro. There is only one oxytocin receptor in humans (OXTR), and it is a G-protein coupled receptor (GPCR) (Gimpl \& Fahrenholz, 2001). We first conducted a dose-response experiment and quantified epicardial cell proliferation at each concentration. As expected, OXT had a concentrationdependent effect on hEpiC proliferation, with a maximal response ( $>2$-fold increase in epicardial cell counts) occurring at $100 \mathrm{nM}$ and an $\mathrm{EC}_{50}$ at $\sim 300 \mathrm{pM}$ (Figure 3A and Supplementary Figure 1A). Gene expression analysis showed that OXTR expression increased 20-30 fold in hEpiCs compared to undifferentiated hiPSCs (Figure 3B). Immunostaining and confocal imaging confirmed that OXTR is present on the cell membrane and in the cytoplasm of hEpiCs (Figure 3C). While the cells clearly expressed OXTR, it should be noted that they did not express OXT as assessed by qRT-PCR (data not shown). In addition to binding its own receptor, oxytocin can also bind to the arginine vasopressin (AVP) receptors in humans, albeit with lower affinity than to OXTR (Gimpl \& Fahrenholz, 2001), however hEpiCs did not express any of the three AVP receptor isoforms (data not shown). These data led us to believe that oxytocin acts through OXTR to induce epicardial activation. We created a knockdown OXTR hiPSC line using an shRNA-lentivirus targeting OXTR. This procedure resulted in a $>90 \%$ knockdown in OXTR expression compared to scrambled epicardial cells (Supplementary Figure 1B) and did not affect the ability of hiPSCs to differentiate into hEpiCs (Supplementary Figure 1C). Exposure to $100 \mathrm{nM}$ OXT led to strong hEpiC activation in scrambled cells, with an even greater response to LIT-001, a recently characterized and very potent non-peptide OXTR agonist (Frantz et al., 2018; Hilfiger et al., 2020) (Supplementary Figure 1D). These findings were confirmed when we stained the cells for proliferation marker Ki67. We observed a $\sim 40 \%$ increase in proliferating hEpiCs in the scrambled line and a slight ( $25 \%)$ decrease in proliferation in the shOXTR line after OXT addition (Figure 3D, E). We next carried out qRT-PCR gene expression analyses to determine if EMT was also affected by OXTR knockdown. We observed a 2 to 4 -fold increase in hEpiC activation (WT1, TCF21, and SNA11 marker expression) in scrambled cells, but these 
effects were entirely prevented in knockdown hEpiCs (Figure $\mathbf{3 F}, \mathbf{G}$ ). We concluded that oxytocin acts through the OXTR to induce human epicardial cell activation in vitro.

Oxytocin acts through the TGF- $\beta$ signaling pathway to induce epicardial activation. To obtain additional clues related to the potential mechanism of action driving the effects of oxytocin, we performed RNA-seq on hEpiCs in control and OXT-treated conditions as described above. RNA was collected for transcriptomic differential gene expression analysis after 3 days of OXT exposure. We found that oxytocin induced significant widespread gene expression changes (Figure 4A). Computational analysis using gene ontology identified upregulated and downregulated clusters consistent with our previous observations on the effects of oxytocin (dedifferentiation to a progenitor-like state, increased proliferation, EMT) (Figure 4B, C). Of particular interest was the upregulation of TGF- $\beta$ /BMP pathway biological processes, as well as developmental ones (Figure 4B). Among downregulated processes, a series of metabolic functions well-ascribed to the epicardium were identified, again consistent with our proposed model of mature epicardial cells becoming epicardial progenitors upon OXT treatment. Leading genes driving the TGF- $\beta$ pathway activation were ligands LEFTY2, GDF15, and INHBB, and the BMP pathway activator TMEM100 (Figure 4D). Overall, these data suggest that oxytocin, through the activation of OXTR, can promote the expression of proteins involved in TGF- $\beta$ and BMP signaling in the epicardium, leading to increased epicardial progenitor cell pools.

Oxytocin signaling plays a role in the formation and migration of the proepicardial organ (PEO) during heart development. Our data suggest that OXT might play an important and overlooked role in epicardial function. We hypothesized that this may be reflected during heart development too, however, very little information is available in this context. We therefore sought to determine if oxtr (the zebrafish ortholog to mammalian OXTR) inhibition would have deleterious effects on epicardium formation during heart development. The epicardium derives from an extra-cardiac cell cluster originating in the splanchnic mesoderm called the proepicardial organ (PEO). Once the PEO forms, it contributes new cells to the developing heart by migration into the epicardial and subepicardial spaces. These cells undergo EMT to form EpiPCs, which go on to differentiate into mature EpiCs and other cardiac cell types (Quijada et al., 2020; Simões \& Riley, 2018). We bred double transgenic myl7DsRed2/tcf21-nls-eGFP zebrafish embryos with a dsRed2 reporter in cardiomyocytes and an enhanced green fluorescent protein (EGFP) tag attached to a nuclear localization signal for tcf21, a well-established EpiPC marker (Kikuchi et al., 2010; J. Wang et al., 2011). This method allowed us to visualize the development of the epicardium and myocardium in real time via fluorescence microscopy. We dissolved different concentrations of atosiban, a competitive OXTR antagonist, into zebrafish embryo medium and imaged epicardium formation at different time points, quantifying the number of tcf21-positive (green) cells covering the myocardium (red) (Figure 5A). We observed a dose-dependent decrease in the number of epicardial cells covering the ventricle (Figure 5B) and atrium (Figure 5C) at 72 hours post-fertilization (hpf), with $100 \mu \mathrm{M}$ atosiban resulting in a maximal decrease of $\sim 2-3$ fold. Thus, oxytocin receptor inhibition significantly disrupts the formation of the epicardium in zebrafish. This delay could be due to defects in proepicardial cell migration to the surface of the developing heart, as it appears that the proepicardial cluster is much farther from the myocardium in the atosiban-treated embryos than in control embryos (Figure 5A). Alternatively, it could be due to a defect in epicardial cell proliferation, as several of our results suggest that OXTR inhibition adversely affects this process in vitro in human cells. 
Oxytocin signaling is necessary for heart regeneration in vivo. The zebrafish is a powerful naturally regenerating organism popular for heart regeneration studies (Aguirre et al., 2014; Jopling et al., 2010; Poss et al., 2002). We decided to test if the role OXT plays in epicardial activation was conserved during zebrafish heart regeneration using a well-established cardiac cryoinjury model (González-Rosa et al., 2011; González-Rosa \& Mercader, 2012) (Figure 6A). After cryoinjury, we observed an 18-fold increase in oxt mRNA levels in zebrafish brains at 3 dpi (days-post injury) and lasting until at least $7 \mathrm{dpi}$ (Figure 6B), suggesting that a burst of oxytocin is released from the brain into the bloodstream after cardiac injury over a prolonged period. To determine the functional significance of this oxytocin burst, we used a specific OXTR inhibitor (L-368,899, a non-peptide OXTR antagonist), and injected it directly into the thoracic cavity of the fish after cryoinjury. The size of the injured myocardial area was assessed by Masson's trichrome staining at different timepoints after injury in control and L368,899 treated hearts. Notably, the difference in scar size between control and treated animals increased as more time passed after cryoinjury. Inhibitor-treated animals displayed a modest increase in injured myocardium ( 1.25-1.5 fold) at 3 and $7 \mathrm{dpi}$ and a $\sim 2$-fold statistically significant increase at $14 \mathrm{dpi}$. In addition, injured hearts treated with L-368,899 had more myocardial fibrosis accumulation than control hearts at $14 \mathrm{dpi}$ (Figure 6E). Taken together, these data suggest that oxytocin signaling is necessary for proper heart regeneration. Because one hallmark of epicardial activation after cardiac injury in vivo is upregulation of WT1 and TCF21 levels (Quijada et al., 2020; Smits et al., 2018), we next collected cryoinjured hearts to quantify the expression of these markers. Both wt $1 \mathrm{~b}$ and tcf21 increased significantly after injury, with peak mRNA levels at 3 dpi (Figure 7A) and expression patterns very consistent with the brain release of oxt (Figure 6B). In addition, immunofluorescence for tcf21 showed an increase in the number of activated epicardial cells at $3 \mathrm{dpi}$, particularly EpiPCs migrating into the damaged tissue (Figure 7B). Intrathoracic L-368,899 injections performed after cryoinjury showed that treated zebrafish hearts had $>2$-fold lower expression of $w t 1 b$ and tcf21 compared to control hearts (Figure 7C). Immunofluorescence for proliferating epicardial cells $\left(\mathrm{h} 3 \mathrm{p}^{+} / \mathrm{wt} 1 \mathrm{~b}^{+}\right)$confirmed this finding, as the percentage of double positive nuclei decreased nearly 2-fold after treatment with L-368,899 (Figure 7D, E). In addition, expression of EMT markers also decreased, as revealed by snai1a and snai2 mRNA levels ( $40-50 \%$ reduction) (Figure 7F). Therefore, oxtr inhibition prevents the robust activation of the epicardium that would normally occur after cardiac injury. We also wondered whether blocking OXT signaling with L-368,899 would have a similar effect on the expansion and proliferation of other cardiac cell types during regeneration, possibly through indirect pathways related to epicardial secretion of pro-regenerative molecules (Lavine et al., 2005; Li et al., 2011). Immunofluorescence for $\mathrm{h} 3 \mathrm{p}$ and myh1e (myosin heavy chain isoform 1E, used as a marker for cardiomyocytes) revealed a $\sim 2$-fold decrease in proliferating CMs after drug treatment (Supplementary Figure 2), thereby recapitulating the effects seen in zebrafish EpiPCs.

\section{DISCUSSION}

Oxytocin is a hypothalamic neuroendocrine hormone best known for its functions in parturition, lactation, and social bonding. However, it also plays other less known physiological roles, including in the cardiovascular system, where it lowers blood pressure, induces negative inotropic and chronotropic effects, and serves as an anti-inflammatory and anti-oxidant (Garrott et al., 2017; Gutkowska et al., 2014; Jankowski et al., 2020). Many of these actions are carried out by OXT-mediated release of atrial natriuretic peptide (ANP) and nitric oxide (NO) (Jankowski et al., 2020), which have well-characterized cardioprotective effects (Jones \& Bolli, 2006; Nishikimi et al., 2006) and may themselves contribute to heart regeneration (Kook et al., 
2003; Rochon et al., 2020). Notably, OXT also induces differentiation of cardiomyocytes from embryonic stem cells (Paquin et al., 2002). Recent studies suggest that damage to neuroendocrine structures, such as the hypothalamus, strongly inhibits endogenous regenerative processes in vertebrates (Zhang et al., 2018), suggesting that critical proregenerative factors are released from this brain region. In addition, acute myocardial infarction activates OXT-releasing neurons in the paraventricular nucleus of the rat hypothalamus (Roy et al., 2019). The above studies, combined with ample evidence to suggest that epicardial activation can be primed by specific signaling factors, such as thymosin $\beta 4$ (Smart et al., 2007, 2011), suggest that oxytocin may be the critical factor in achieving sufficient EpiPC activation and differentiation to regenerate the lost myocardium after cardiac injury.

Here, we have shown that OXT induces a pro-regenerative phenotype in vitro. When maturelike hEpiCs are exposed to $100 \mathrm{nM} \mathrm{OXT}$, they increase their proliferation rates (Figure 2B-D) and adopt an embryonic gene expression profile (Figure 2F), two hallmarks of epicardial activation. Notably, oxytocin induces an even greater proliferative response than thymosin $\beta 4$ (Figure 2E). It appears as though the peptide acts through its receptor to exert its effects as the increases in hEpiC proliferation and activation that are seen in scrambled cells are completely abolished, if not reversed, in OXTR knockdown epicardial cells (Figure 3D-G). Although OXT is also able to bind to AVP receptors with lower affinity (Gimpl \& Fahrenholz, 2001), it does not appear as if this signaling pathway plays a role in our model, as hEpiCs do not even express these receptors. It is also likely that the pro-regenerative effects of oxytocin are not just limited to one cardiac cell type, as we found similar increases in proliferation when we exposed hiPSC-derived cardiomyocytes to OXT (data not shown), suggesting that it has a global effect on heart regeneration. Although our findings using two dimensional monolayers are encouraging, we acknowledge that producing similar results with three dimensional cardiac organoids would be of even greater translational value. To that end, current efforts are utilizing our recently described human heart organoid $(\mathrm{hHO})$ model to elucidate the effects of oxytocin signaling on epicardial development and regeneration in a "miniature heart". These 3D structures self-organize into beating spheroids complete with cardiac-chamber like features, the entire array of cell types found in the heart, vascularization, and cardiac functional activity (Lewis-Israeli, Volmert, et al., 2021; Lewis-Israeli, Wasserman, et al., 2021). We have successfully differentiated our OXTR knockdown hiPSCs to hHOs and are exploring multiple injury and toxicity models, including cryoinjury, hypoxia induction, and anthracycline-induced cardiotoxicity. While preliminary results suggest that oxytocin signaling is required for proper epicardial activation after $\mathrm{hHO}$ cryoinjury (data not shown), we will continue to characterize the effects on epicardial cell and cardiomyocyte proliferation and migration. We will also study the fibrotic injury response by cryosectioning and staining the organoids to fully elucidate the effects of OXTR knockdown on cardiac regeneration in a dish.

In order to further demonstrate the importance of oxytocin signaling to heart regeneration, we have also applied our findings to zebrafish, one of the most powerful naturally regenerating animal models known (Beffagna, 2019; Jopling et al., 2010; Poss et al., 2002). Several seminal studies of the last $\sim 20$ years have shown that the epicardium plays a crucial role in zebrafish heart regeneration (Cao et al., 2016; Cao \& Poss, 2018; Kikuchi et al., 2011; Lepilina et al., 2006), therefore it is the perfect model system with which to expand our studies in vivo. Here, we have demonstrated that pharmacological inhibition of OXT signaling significantly slows cardiac regeneration after cryoinjury, as seen by increased fibrosis accumulation and myocardial injury area when zebrafish are treated with L-368,899 (Figure 6C-E). In addition, 
OXTR inhibition prevents the proliferative response of both epicardial cells and cardiomyocytes that is normally seen after cryoinjury (Figure 7D, E and Supplementary Figure 2). Future studies will explore the effects of OXTR inhibition on other cardiac cell types, including endothelial cells, cardiac fibroblasts, and immune cells. Our data also suggest that OXT signaling is required for proper epicardial activation, as L-368,899 treatment decreases the cardiac expression of several genes that are involved in EpiPC expansion and EMT (Figure 7C, F). Although this antagonist is specific for the oxytocin receptor (Pettibone et al., 1993), in order to fully demonstrate that the above phenotype is not due to off-target effects, we intend to generate an oxtr knockout zebrafish line through CRISPR-Cas9. To accomplish this task, we will utilize a "codon-optimized" Tol2 transposase-based system to ensure that the CRISPR reagents are effectively delivered into the host genome (Kawakami, 2007; Kwan et al., 2007; Mackey et al., 2020). Growing the knockout animals to adulthood and subsequently performing cryoinjuries will allow us to study the effects of oxytocin signaling on zebrafish heart regeneration with even greater precision.

Our hypothesis is that oxytocin is released from the brain into the bloodstream after cardiac injury to facilitate epicardial activation and heart regeneration. Here, we have demonstrated that oxt mRNA levels increase 20 -fold in zebrafish brains 3 days after cardiac cryoinjury (Figure 6B), with a corresponding increase seen in wt $1 \mathrm{~b}$ and tcf21 expression in the injured hearts (Figure 7A). While this is an encouraging correlational finding, it is possible that the increase in epicardial activation could be due to local effects, as the heart is able to produce some OXT on its own (Gutkowska et al., 2000; Jankowski et al., 1998). In order to confirm that our results are due to hypothalamic OXT release, we could utilize the Nitroreductase/Metronidazole (NTR/Mtz) system (Curado et al., 2007, 2008) to selectively ablate cells of paraventricular nucleus (PVN), the main site of OXT production. By preventing the brain from synthesizing oxytocin, we could more thoroughly elucidate how important systemic hormone release is to heart regeneration. However, it should be noted that after cardiac cryoinjury, the zebrafish brain increases its expression of oxt 10-fold when treated with L-368,899, suggesting a compensatory OXT release (data not shown). If this is truly the case, and heart regeneration is still adversely affected after OXTR inhibition, then it is likely that the epicardial regeneration process depends on brain-derived oxytocin to a large extent. It will also be necessary to confirm that this oxt mRNA is indeed translated to protein and released into circulation, so future efforts will utilize ELISA assays to detect OXT peptide in cryoinjured zebrafish blood samples. In addition, we will also collect media samples from hHOs after cryoinjury and use ELISA or LC-MS to directly quantify OXT levels.

In conclusion, we show here that oxytocin is an important activator of EpiPCs and is necessary for heart regeneration and proper epicardium development. We demonstrate that OXT induces reprogramming of human epicardial cells into an epicardial progenitor state by activating the OXTR. These effects are mediated through the TGF- $\beta$ pathway and its associated downstream effectors. Overall, our results establish a previously uncharacterized link between OXT release after cardiac injury and heart regeneration and provide evidence to support the notion that cardiac regeneration is, at least partially, under neuroendocrine control. These findings offer exciting translational potential, as oxytocin or one of its agonists could be used in the clinic to aid in the recovery from severe cardiac events, such as an $\mathrm{Ml}$, and prevent progression to heart failure in the future.

\section{AUTHOR CONTRIBUTIONS}


A.H.W. and A.A. designed all experiments and conceptualized the work. A.H.W. performed all experiments and data analysis. M.V. performed zebrafish experiments. A.R.H. performed cell culture and zebrafish husbandry and experiments. Y.R.L.-I. acquired in vitro data. M.D.D. and A.L.M. acquired and analyzed molecular biology data. A.H.W. prepared all figures and A.H.W. and A.A. wrote the manuscript.

\section{ACKNOWLEDGEMENTS}

We wish to thank the MSU Genomics Core for RNA sequencing support and Dr. William Jackson at the MSU Department of Pharmacology/Toxicology for access to confocal microscopes. We also want to thank all members of the Aguirre lab for helpful comments and discussions. Work in Dr. Aguirre's laboratory was supported by the National Heart, Lung, and Blood Institute of the National Institutes of Health under award numbers K01HL135464, R01HL151505, by the American Heart Association under award number 19 IPLOI34660342 and by the Spectrum-MSU Foundation.

\section{DISCLOSURES}

The authors declare no conflicts of interest. 


\section{REFERENCES}

Aguirre, A., Montserrat, N., Zacchigna, S., Nivet, E., Hishida, T., Krause, M. N., Kurian, L., Ocampo, A., Vazquez-Ferrer, E., Rodriguez-Esteban, C., Kumar, S., Moresco, J. J., Yates, J. R., Campistol, J. M., Sancho-Martinez, I., Giacca, M., \& Izpisua Belmonte, J. C. (2014). In vivo activation of a conserved microRNA program induces mammalian heart regeneration. Cell Stem Cell, 15(5), 589-604. https://doi.org/10.1016/j.stem.2014.10.003

Bao, X., Lian, X., Hacker, T. A., Schmuck, E. G., Qian, T., Bhute, V. J., Han, T., Shi, M., Drowley, L., Plowright, A. T., Wang, Q. D., Goumans, M. J., \& Palecek, S. P. (2016). Long-term self-renewing human epicardial cells generated from pluripotent stem cells under defined xeno-free conditions. Nature Biomedical Engineering, 1, 1-12. https://doi.org/10.1038/s41551-016-0003

Bao, X., Lian, X., Qian, T., Bhute, V. J., Han, T., \& Palecek, S. P. (2017). Directed differentiation and long-term maintenance of epicardial cells derived from human pluripotent stem cells under fully defined conditions. Nature Protocols, 12(9), 1890-1900. https://doi.org/10.1038/nprot.2017.080

Beffagna, G. (2019). Zebrafish as a Smart Model to Understand Regeneration After Heart Injury: How Fish Could Help Humans. Frontiers in Cardiovascular Medicine, 6(August), 18. https://doi.org/10.3389/fcvm.2019.00107

Bergmann, O., Bhardwaj, R. D., Bernard, S., Zdunek, S., Walsh, S., Zupicich, J., Alkass, K., Buchholz, B. A., Jovinge, S., Frisén, J., Bergmann, O., Bhardwaj, R. D., Bernard, S., Zdunek, S., Barnabi-heider, F., Walsh, S., Zupicich, J., Alkass, K., Buchholz, B. A., ... Frisen, J. (2009). Evidence for Cardiomyocyte Renewal in Humans. Science, 324(5923), 98-102.

Bise, T., \& Jaźwińska, A. (2019). Intrathoracic injection for the study of adult zebrafish heart. Journal of Visualized Experiments, 147, 1-10. https://doi.org/10.3791/59724

Cai, C.-L., Martin, J. C., Sun, Y., Cui, L., Wang, L., Ouyang, J., Yang, L., Bu, L., Xingqun, L., Zhang, X., Stallcup, W. B., Denton, C. P., McCulloch, A., Chen, J., \& Evans, S. M. (2008). A myocardial lineage derives from Tbx18 epicardial cells. Nature, 454(7200), 104-108. https://doi.org/10.1038/nature06969.A

Cao, J., Navis, A., Cox, B. D., Dickson, A. L., Gemberling, M., Karra, R., Bagnat, M., \& Poss, K. D. (2016). Single epicardial cell transcriptome sequencing identifies caveolin 1 as an essential factor in zebrafish heart regeneration. Development (Cambridge), 143, 232-243. https://doi.org/10.1242/dev.130534

Cao, J., \& Poss, K. D. (2018). The epicardium as a hub for heart regeneration. Nature Reviews Cardiology, 15(10), 631-647. https://doi.org/10.1038/s41569-018-0046-4

Curado, S., Anderson, R. M., Jungblut, B., Mumm, J., Schroeter, E., \& Stainier, D. Y. R. (2007). Conditional targeted cell ablation in zebrafish: A new tool for regeneration studies. Developmental Dynamics, 236(4), 1025-1035. https://doi.org/10.1002/dvdy.21100 
Curado, S., Stainier, D. Y. R., \& Anderson, R. M. (2008). Nitroreductase-mediated cell/tissue ablation in zebrafish: A spatially and temporally controlled ablation method with applications in developmental and regeneration studies. Nature Protocols, 3(6), 948-954. https://doi.org/10.1038/nprot.2008.58

Dampney, R. A. L. (2016). Central neural control of the cardiovascular system: Current perspectives. Advances in Physiology Education, 40(3), 283-296.

https://doi.org/10.1152/advan.00027.2016

Fan, D., Takawale, A., Lee, J., \& Kassiri, Z. (2012). Cardiac fibroblasts, fibrosis and extracellular matrix remodeling in heart disease. Fibrogenesis \& Tissue Repair, 5, 1-13. http://www.fibrogenesis.com/content/5/1/15\%5Cnhttp://ovidsp.ovid.com/ovidweb.cgi?T=JS \&PAGE $=$ reference\&D=emed10\&NEWS $=N \& A N=2012547739$

Frantz, M. C., Pellissier, L. P., Pflimlin, E., Loison, S., Gandiá, J., Marsol, C., Durroux, T., Mouillac, B., Becker, J. A. J., Le Merrer, J., Valencia, C., Villa, P., Bonnet, D., \& Hibert, M. (2018). LIT-001, the First Nonpeptide Oxytocin Receptor Agonist that Improves Social Interaction in a Mouse Model of Autism. Journal of Medicinal Chemistry, 61(19), 86708692. https://doi.org/10.1021/acs.jmedchem.8b00697

Garrott, K., Dyavanapalli, J., Cauley, E., Dwyer, M. K., Kuzmiak-Glancy, S., Wang, X., Mendelowitz, D., \& Kay, M. W. (2017). Chronic activation of hypothalamic oxytocin neurons improves cardiac function during left ventricular hypertrophy-induced heart failure. Cardiovascular Research, 113(11), 1318-1328. https://doi.org/10.1093/cvr/cvx084

Gimpl, G., \& Fahrenholz, F. (2001). The oxytocin receptor system: Structure, function, and regulation. Physiological Reviews, 81(2), 629-683.

https://doi.org/10.1152/physrev.2001.81.2.629

González-Rosa, J. M., Martín, V., Peralta, M., Torres, M., \& Mercader, N. (2011). Extensive scar formation and regression during heart regeneration after cryoinjury in zebrafish. Development, 138(9), 1663-1674. https://doi.org/10.1242/dev.060897

González-Rosa, J. M., \& Mercader, N. (2012). Cryoinjury as a myocardial infarction model for the study of cardiac regeneration in the zebrafish. Nature Protocols, 7(4), 782-788. https://doi.org/10.1038/nprot.2012.025

Gordan, R., Gwathmey, J. K., \& Xie, L.-H. (2015). Autonomic and endocrine control of cardiovascular function. World Journal of Cardiology, 7(4), 204-214. https://doi.org/10.4330/wjc.v7.i4.204

Gutkowska, J., Jankowski, M., \& Antunes-Rodrigues, J. (2014). The role of oxytocin in cardiovascular regulation. Brazilian Journal of Medical and Biological Research, 47(3), 206-214. https://doi.org/10.1590/1414-431X20133309

Gutkowska, J., Jankowski, M., Mukaddam-Daher, S., \& McCann, S. M. (2000). Oxytocin is a cardiovascular hormone. Brazilian Journal of Medical and Biological Research, 33(6), 625-633. https://doi.org/10.1590/S0100-879X2000000600003

Guyenet, P. G., \& Bayliss, D. A. (2015). Neural Control of Breathing and CO2 Homeostasis. Neuron, 87(5), 946-961. https://doi.org/10.1016/j.neuron.2015.08.001

Hilfiger, L., Zhao, Q., Kerspern, D., Inquimbert, P., Andry, V., Goumon, Y., Darbon, P., Hibert, M., \& Charlet, A. (2020). A Nonpeptide Oxytocin Receptor Agonist for a Durable Relief of Inflammatory Pain. Scientific Reports, 10(1), 1-10. https://doi.org/10.1038/s41598-02059929-w

Hirose, K., Payumo, A. Y., Cutie, S., Hoang, A., Zhang, H., Guyot, R., Lunn, D., Bigley, R. B., Yu, H., Wang, J., Smith, M., Gillett, E., Muroy, S. E., Schmid, T., Wilson, E., Field, K. A., Reeder, D. A. M., Maden, M., Yartsev, M. M., ... Huang, G. N. (2019). Evidence for hormonal control of heart regenerative capacity during endothermy acquisition. Science, 364(6436), 184-188. https://doi.org/10.1126/science.aar2038 
Jankowski, M., Broderick, T. L., \& Gutkowska, J. (2020). The Role of Oxytocin in Cardiovascular Protection. Frontiers in Psychology, 11, 1-17.

https://doi.org/10.3389/fpsyg.2020.02139

Jankowski, M., Hajjar, F., Al Kawas, S., Mukaddam-Daher, S., Hoffman, G., Mccann, S. M., \& Gutkowska, J. (1998). Rat heart: A site of oxytocin production and action. Proceedings of the National Academy of Sciences of the United States of America, 95(24), 14558-14563. https://doi.org/10.1073/pnas.95.24.14558

Jones, S. P., \& Bolli, R. (2006). The ubiquitous role of nitric oxide in cardioprotection. Journal of Molecular and Cellular Cardiology, 40(1), 16-23.

https://doi.org/10.1016/j.yjmcc.2005.09.011

Jopling, C., Sleep, E., Raya, M., Marti, M., Raya, A., \& Izpisua Belmonte, J. C. (2010). Zebrafish heart regeneration occurs by cardiomyocyte dedifferentiation and proliferation. Nature, 176(12), 139-148. https://doi.org/10.1038/nature08899.Zebrafish

Kawakami, K. (2007). Tol2: A versatile gene transfer vector in vertebrates. Genome Biology, 8(SUPPL 1), 1-10. https://doi.org/10.1186/gb-2007-8-s1-s7

Kikuchi, K., Holdway, J. E., Major, R. J., Blum, N., Dahn, R. D., Begemann, G., \& Poss, K. D. (2011). Retinoic Acid Production by Endocardium and Epicardium Is an Injury Response Essential for Zebrafish Heart Regeneration. Developmental Cell, 20(3), 397-404. https://doi.org/10.1016/j.devcel.2011.01.010

Kikuchi, K., Holdway, J. E., Werdich, A. A., Anderson, R. M., Fang, Y., Egnaczyk, G. F., Evans, T., MacRae, C. A., Stainier, D. Y. R., \& Poss, K. D. (2010). Primary contribution to zebrafish heart regeneration by gata4+ cardiomyocytes. Nature, 464(7288), 601-605. https://doi.org/10.1038/nature08804

Kook, H., Itoh, H., Choi, B. S., Sawada, N., Doi, K., Hwang, T. J., Kim, K. K., Arai, H., Baik, Y. H., \& Nakao, K. (2003). Physiological concentration of atrial natriuretic peptide induces endothelial regeneration in vitro. American Journal of Physiology - Heart and Circulatory Physiology, 284(4), 1388-1397. https://doi.org/10.1152/ajpheart.00414.2002

Kwan, K. M., Fujimoto, E., Grabher, C., Mangum, B. D., Hardy, M. E., Campbell, D. S., Parant, J. M., Yost, H. J., Kanki, J. P., \& Chien, C. Bin. (2007). The Tol2kit: A multisite gatewaybased construction Kit for Tol2 transposon transgenesis constructs. Developmental Dynamics, 236(11), 3088-3099. https://doi.org/10.1002/dvdy.21343

Laflamme, M. A., \& Murry, C. E. (2011). Heart Regeneration. Nature, 473(7347), 326-335. https://doi.org/10.1038/nature10147. Heart

Lavine, K. J., Yu, K., White, A. C., Zhang, X., Smith, C., Partanen, J., \& Ornitz, D. M. (2005). Endocardial and epicardial derived FGF signals regulate myocardial proliferation and differentiation in vivo. Developmental Cell, 8(1), 85-95.

https://doi.org/10.1016/j.devcel.2004.12.002

Lepilina, A., Coon, A. N., Kikuchi, K., Holdway, J. E., Roberts, R. W., Burns, C. G., \& Poss, K. D. (2006). A Dynamic Epicardial Injury Response Supports Progenitor Cell Activity during Zebrafish Heart Regeneration. Cell, 127(3), 607-619.

https://doi.org/10.1016/j.cell.2006.08.052

Lewis-Israeli, Y. R., Volmert, B. D., Gabalski, M. A., Huang, A. R., \& Aguirre, A. (2021). Generating Self-Assembling Human Heart Organoids Derived from Pluripotent Stem Cells. Journal of Visualized Experiments, 175, 1-18. https://doi.org/10.3791/63097

Lewis-Israeli, Y. R., Wasserman, A. H., Gabalski, M. A., Volmert, B. D., Ming, Y., Ball, K. A., Yang, W., Zou, J., Ni, G., Pajares, N., Chatzistavrou, X., Li, W., Zhou, C., \& Aguirre, A. (2021). Self-assembling human heart organoids for the modeling of cardiac development and congenital heart disease. Nature Communications, 12(1), 1-16. https://doi.org/10.21203/rs.3.rs-132349/v1 
Li, P., Cavallero, S., Gu, Y., Chen, T. H. P., Hughes, J., Hassan, A. B., Brüning, J. C., Pashmforoush, M., \& Sucov, H. M. (2011). IGF signaling directs ventricular cardiomyocyte proliferation during embryonic heart development. Development, 138(9), 1795-1805. https://doi.org/10.1242/dev.054338

Mackey, A. S., Redd, P. S., DeLaurier, A., \& Hancock, C. N. (2020). Codon optimized Tol2 transposase results in increased transient expression of a crystallin-GFP transgene in zebrafish. MicroPublication Biology, 2020, 2011-2014. https://doi.org/10.17912/micropub.biology.000268

Morrison, S. F. (2016). Central control of body temperature. F1000Research, 5, 1-10. https://doi.org/10.12688/F1000RESEARCH.7958.1

Nishikimi, T., Maeda, N., \& Matsuoka, H. (2006). The role of natriuretic peptides in cardioprotection. Cardiovascular Research, 69(2), 318-328. https://doi.org/10.1016/j.cardiores.2005.10.001

Palma, J.-A., \& Benarroch, E. E. (2014). Neural control of the heart: recent concepts and clinical correlations. Neurology, 83(3), 261-271.

Paquin, J., Danalache, B. A., Jankowski, M., McCann, S. M., \& Gutkowska, J. (2002). Oxytocin induces differentiation of P19 embryonic stem cells to cardiomyocytes. Proceedings of the National Academy of Sciences of the United States of America, 99(14), 9550-9555. https://doi.org/10.1073/pnas.152302499

Peralta, M., González-Rosa, J. M., Marques, I. J., \& Mercader, N. (2014). The epicardium in the embryonic and adult zebrafish. Journal of Developmental Biology, 2(2), 101-116. https://doi.org/10.3390/jdb2020101

Pettibone, D. J., Clineschmidt, B. V., Guidotti, M. T., Lis, E. V., Reiss, D. R., Woyden, C. J., Bock, M. G., Evans, B. E., Freidinger, R. M., Hobbs, D. W., Veber, D. F., Williams, P. D., Chiu, S. -H L., Thompson, K. L., Schorn, T. W., Siegl, P. K. S., Kaufman, M. J., Cukierski, M. A., Haluska, G. J., ... Novy, M. J. (1993). L-368,899, a potent orally active oxytocin antagonist for potential use in preterm labor. Drug Development Research, 30(3), 129142. https://doi.org/10.1002/ddr.430300305

Poss, K. D., Wilson, L. G., \& Keating, M. T. (2002). Heart regeneration in zebrafish. Science, 298(5601), 2188-2190. https://doi.org/10.1126/science.1077857

Quijada, P., Trembley, M. A., \& Small, E. M. (2020). The Role of the Epicardium during Heart Development and Repair. Circulation Research, 126, 377-394. https://doi.org/10.1161/CIRCRESAHA.119.315857

Rao, K. S., \& Spees, J. L. (2017). Harnessing Epicardial Progenitor Cells and Their Derivatives for Rescue and Repair of Cardiac Tissue After Myocardial Infarction. Curr Mol Biol Rep, 3(3), 149-158. https://doi.org/10.1016/j.physbeh.2017.03.040

Rhee, S. S., \& Pearce, E. N. (2011). The Endocrine System and the Heart: A Review. Revista Española de Cardiología (English Edition), 64(3), 220-231. https://doi.org/10.1016/j.rec.2010.10.016

Riley, P. R. (2012). An Epicardial Floor Plan for Building and Rebuilding the Mammalian Heart. Current Topics in Developmental Biology, 100, 233-251. https://doi.org/10.1016/B978-012-387786-4.00007-5

Rochon, E. R., Missinato, M. A., Xue, J., Tejero, J., Tsang, M., Gladwin, M. T., \& Corti, P. (2020). Nitrite Improves Heart Regeneration in Zebrafish. Antioxidants and Redox Signaling, 32(6), 363-377. https://doi.org/10.1089/ars.2018.7687

Roh, E., Song, D. K., \& Kim, M. S. (2016). Emerging role of the brain in the homeostatic regulation of energy and glucose metabolism. Experimental and Molecular Medicine, 48(3), 1-12. https://doi.org/10.1038/emm.2016.4

Roy, R. K., Augustine, R. A., Brown, C. H., \& Schwenke, D. O. (2019). Acute myocardial 
infarction activates magnocellular vasopressin and oxytocin neurones. Journal of Neuroendocrinology, 31(12), 1-10. https://doi.org/10.1111/jne.12808

Sallin, P., \& Jaźwińska, A. (2016). Acute stress is detrimental to heart regeneration in zebrafish. Open Biology, 6, 1-15. https://doi.org/10.1098/rsob.160012

Simões, F. C., \& Riley, P. R. (2018). The ontogeny, activation and function of the epicardium during heart development and regeneration. Development (Cambridge), 145(7). https://doi.org/10.1242/dev.155994

Smart, N., Bollini, S., Dubé, K. N., Vieira, J. M., Zhou, B., Davidson, S., Yellon, D., Riegler, J., Price, A. N., Lythgoe, M. F., Pu, W. T., \& Riley, P. R. (2011). De novo cardiomyocytes from within the activated adult heart after injury. Nature, 474(7353), 640-644. https://doi.org/10.1038/nature10188

Smart, N., Risebro, C. A., Melville, A. A. D., Moses, K., Schwartz, R. J., Chien, K. R., \& Riley, P. R. (2007). Thymosin $\beta 4$ induces adult epicardial progenitor mobilization and neovascularization. Nature, 445(7124), 177-182. https://doi.org/10.1038/nature05383

Smits, A. M., Dronkers, E., \& Goumans, M. J. (2018). The epicardium as a source of multipotent adult cardiac progenitor cells: Their origin, role and fate. Pharmacological Research, 127, 129-140. https://doi.org/10.1016/j.phrs.2017.07.020

Uygur, A., \& Lee, R. T. (2016). Mechanisms of Cardiac Regeneration. Developmental Cell, 36(4), 362-374. https://doi.org/10.1016/j.devcel.2016.01.018

Virani, S. S., Alonso, A., Benjamin, E. J., Bittencourt, M. S., Callaway, C. W., Carson, A. P., Chamberlain, A. M., Chang, A. R., Cheng, S., Delling, F. N., Djousse, L., Elkind, M. S. V., Ferguson, J. F., Fornage, M., Khan, S. S., Kissela, B. M., Knutson, K. L., Kwan, T. W., Lackland, D. T., ... Heard, D. G. (2020). Heart disease and stroke statistics-2020 update: A report from the American Heart Association. Circulation, 141, E139-E596. https://doi.org/10.1161/CIR.0000000000000757

Wang, J., Panáková, D., Kikuchi, K., Holdway, J. E., Gemberling, M., Burris, J. S., Singh, S. P., Dickson, A. L., Lin, Y. F., Khaled Sabeh, M., Werdich, A. A., Yelon, D., MacRae, C. A., \& Poss, K. D. (2011). The regenerative capacity of zebrafish reverses cardiac failure caused by genetic cardiomyocyte depletion. Development, 138(16), 3421-3430. https://doi.org/10.1242/dev.068601

Wang, Y. L., Yu, S. N., Shen, H. R., Wang, H. J., Wu, X. P., Wang, Q. L., Zhou, B., \& Tan, Y. Z. (2021). Thymosin $\beta 4$ released from functionalized self-assembling peptide activates epicardium and enhances repair of infarcted myocardium. Theranostics, 11(9), 42624280. https://doi.org/10.7150/THNO.52309

Wei, K., Serpooshan, V., Hurtado, C., Diez-Cunado, M., Zhao, M., Maruyama, S., Zhu, W., Fajardo, G., Noseda, M., Nakamura, K., Tian, X., Liu, Q., Wang, A., Matsuura, Y., Bushway, P., Cai, W., Savchenko, A., Mahmoudi, M., Schneider, M. D., ... Ruiz-Lozano, P. (2015). Epicardial FSTL1 reconstitution regenerates the adult mammalian heart. Nature, 525(7570), 479-485. https://doi.org/10.1038/nature15372

Xu, S., Xie, F., Tian, L., Fallah, S., Babaei, F., Manno, S. H. C., Manno, F. A. M., Zhu, L., Wong, K. F., Liang, Y., Ramalingam, R., Sun, L., Wang, X., Plumb, R., Gethings, L., Lam, Y. W., \& Cheng, S. H. (2020). Estrogen accelerates heart regeneration by promoting the inflammatory response in zebrafish. Journal of Endocrinology, 245(1), 39-51. https://doi.org/10.1530/JOE-19-0413

Zhang, M., Chen, Y., Xu, H., Yang, L., Yuan, F., Li, L., Xu, Y., Chen, Y., Zhang, C., \& Lin, G. (2018). Melanocortin Receptor 4 Signaling Regulates Vertebrate Limb Regeneration. Developmental Cell, 46(4), 397-409. https://doi.org/10.1016/j.devcel.2018.07.021

Zhou, B., Honor, L. B., He, H., Qing, M., Oh, J. H., Butterfield, C., Lin, R. Z., Melero-Martin, J. M., Dolmatova, E., Duffy, H. S., Von Gise, A., Zhou, P., Hu, Y. W., Wang, G., Zhang, B., 
Wang, L., Hall, J. L., Moses, M. A., McGowan, F. X., \& Pu, W. T. (2011). Adult mouse epicardium modulates myocardial injury by secreting paracrine factors. Journal of Clinical Investigation, 121(5), 1894-1904. https://doi.org/10.1172/JCl45529

Zhou, B., Ma, Q., Rajagopal, S., Wu, S. M., Domian, I., Rivera-Feliciano, J., Jiang, D., Von Gise, A., Ikeda, S., Chien, K. R., \& Pu, W. T. (2008). Epicardial progenitors contribute to the cardiomyocyte lineage in the developing heart. Nature, 454(7200), 109-113. https://doi.org/10.1038/nature07060

\section{FIGURE LEGENDS}

Figure 1: Differentiation of Mature-Like Epicardial Cell Cultures from hiPSCs. A) Schematic of protocol used for hEpiC differentiation. B) Brightfield images of hEpiCs through four passages showing the gradual accumulation of the classic epithelial cell "cobblestone" morphology. C) qRT-PCR data for epicardial markers WT1 and TCF21 in hiPSCs and hEpiCs. D-E) Confocal immunofluorescent images (D) and quantification (E) showing nearly $100 \%$ epicardial differentiation efficiency from hiPSCs. In (D), epicardial cells are labeled with TCF21 (green), nuclei with DAPI (blue); $n=10$ images, scale bar: 100 $\mu \mathrm{m}$. F-G) Time course qRT-PCR data for WT1 (F) and CDH1 (G) throughout hEpiC differentiation, suggesting an increase in epicardial nature over time. H) Absolute counts of DAPI-labeled nuclei after exposure to different combinations of Vitamin C and SB431542. I) qRT-PCR data for hEpiCs in the presence or absence of SB431542 (SB), showing an increase in maturity when this compound is removed from the media.

Figure 2: Oxytocin Induces Proliferation and Activation of hiPSC-Derived Epicardial Cells. A) Screening results for 15 candidate neuroendocrine peptides, shown as the percentage of hEpiCs expressing H3P after exposure to each compound. B-C) Confocal immunofluorescent images (B) and quantification (C) of proliferating hEpiCs after 3 day OXT exposure. In (B), epicardial cells are labeled with WT1 (red), epithelial membranes are labeled with TJP1 (magenta), proliferating cells (arrowheads) are labeled with Ki67 (green), nuclei are labeled with DAPI (blue); n=10 images, scale bar: $25 \mu \mathrm{m}$. D-E) Absolute counts of DAPI-labeled epicardial cell nuclei after 3 day exposure to OXT (D) or thymosin $\beta 4$ (E), a compound previously shown to induce epicardial activation. F) qRT-PCR data for epicardial cells exposed to OXT, showing an increase in EpiPC (WT1, TCF21), EMT (SNA11), and mesenchymal (NT5E) markers, without a corresponding increase in epithelial $(C D H 1)$ markers.

Figure 3: OXTR Knockdown Decreases Proliferation and Activation of hiPSC-Derived Epicardial Cells. A) Dose-response data for hEpiCs exposed to different concentrations of OXT over the course of 3 days, expressed as number of nuclei at each concentration. B) Time course qRT-PCR data for OXTR in hEpiCs. C) Confocal immunofluorescent images for OXTR (green) and DAPI (blue), showing oxytocin receptor expression on the cell membrane and in the peri-nuclear region; Scale bar: $25 \mu \mathrm{m}$. DE) Confocal immunofluorescent images (D) and quantification (E) of proliferating hEpiCs in both cell lines in the presence and absence of OXT at day 25 of differentiation. In (D), epicardial cells are labeled with WT1 (red), epithelial membranes are labeled with TJP1 (magenta), proliferating cells are labeled with Ki67 (green), nuclei are labeled with DAPI (blue); $n=10$ images, scale bar: $50 \mu \mathrm{m}$. F-G) qRT-PCR data for scrambled $(F)$ and shOXTR $(G)$ hEpiCs, showing an increase in epicardial activation in control cells that is prevented after OXTR knockdown. V: vehicle.

Figure 4: Transcriptome Analysis of OXT-Treated Epicardial Cells. A) Volcano plot showing differentially expressed genes between control and OXT-treated hEpiCs, as determined by RNA sequencing; Blue dots correspond to genes with a fold change $\geq 1.4$ and a $p$-value $\leq 0.05$. B-C) Gene ontology analysis showing upregulated and downregulated biological processes (B) and molecular functions (C) after oxytocin exposure in hEpiCs. D) Relative mRNA expression of ligands for the TGF- $\beta$ pathway and activators of BMP signaling in control and OXT-treated hEpiCs. 
Figure 5: Oxytocin Signaling is Necessary for Proper Epicardium Development. A-C) Fluorescent images (A) and epicardial cell counts per heart chamber (B-C) of developing zebrafish embryos at 72 hpf treated with different concentrations of atosiban. In (A), proepicardial and epicardial cells are labeled with GFP (green dots, arrowheads), myocardium is labeled with DsRed2 (red), dashed lines demarcate proepicardial organ; $n \geq 8$ embryos per condition; A: Atrium, PE: Proepicardium, V: Ventricle.

Figure 6: Oxytocin Signaling is Necessary for Proper Heart Regeneration. A) Example of a freshly cryoinjured zebrafish heart, dashed lines demarcate injured area. B) Time course qRT-PCR data for oxt in zebrafish brains after cardiac cryoinjury. C-D) Masson's trichrome staining (C) and quantification (D) of cryoinjured zebrafish hearts at 3, 7, and 14 dpi treated with and without $1 \mu \mathrm{M} \mathrm{L-368,899.} \mathrm{In} \mathrm{(C),}$ myocardium is stained red, collagen is stained blue, dashed lines demarcate injured area, which is quantified in (D); $n=3-5$ hearts per time point. E) Representative images of cryoinjured zebrafish myocardium (red) at $14 \mathrm{dpi}$ showing more fibrosis accumulation (blue) after OXTR inhibition; BA: Bulbus Arteriosus, IA: Injured Area, V: Ventricle.

Figure 7: Inhibition of Oxytocin Signaling Prevents Epicardial Activation After Cardiac Injury. A) Time course qRT-PCR data for $w t 1 b$ and $t c f 21$ in zebrafish hearts after cardiac cryoinjury; ${ }^{\star} P<0.05$ versus sham operated heart. B) Confocal immunofluorescent images of cryoinjured zebrafish hearts at 3 dpi showing epicardial activation after cardiac injury. Epicardial cells are labeled with TCF21 (green), cardiomyocytes are labeled with TNNT2 (red), nuclei are labeled with DAPI (blue); First images are low magnification (scale bar: $50 \mu \mathrm{m}$ ), last 4 images are high magnification of the area demarcated by the dashed white box (scale bar: $25 \mu \mathrm{m}$ ). C) qRT-PCR data for wt1b and tcf21 in cryoinjured zebrafish hearts at 3 dpi treated with and without $1 \mu \mathrm{M}$ L-368,899 (L-368). D-E) Confocal immunofluorescent images (D) and quantification (E) of proliferating epicardial cells in cryoinjured zebrafish hearts 3 days after cardiac cryoinjury. In (D), epicardial cells are labeled with WT1 (red), proliferating cells are labeled with H3P (green), nuclei are labeled with DAPI (blue); First images are low magnification (scale bar: 50 $\mu \mathrm{m}$ ), last 4 images are high magnification of the area demarcated by the dashed white box (scale bar: $25 \mu \mathrm{m}) ; \mathrm{n}=8$ images. F) qRT-PCR data for snai1a and snai2 in cryoinjured zebrafish hearts at 3 dpi treated with and without $1 \mu \mathrm{M} \mathrm{L-368,899}$ (L-368). 
A

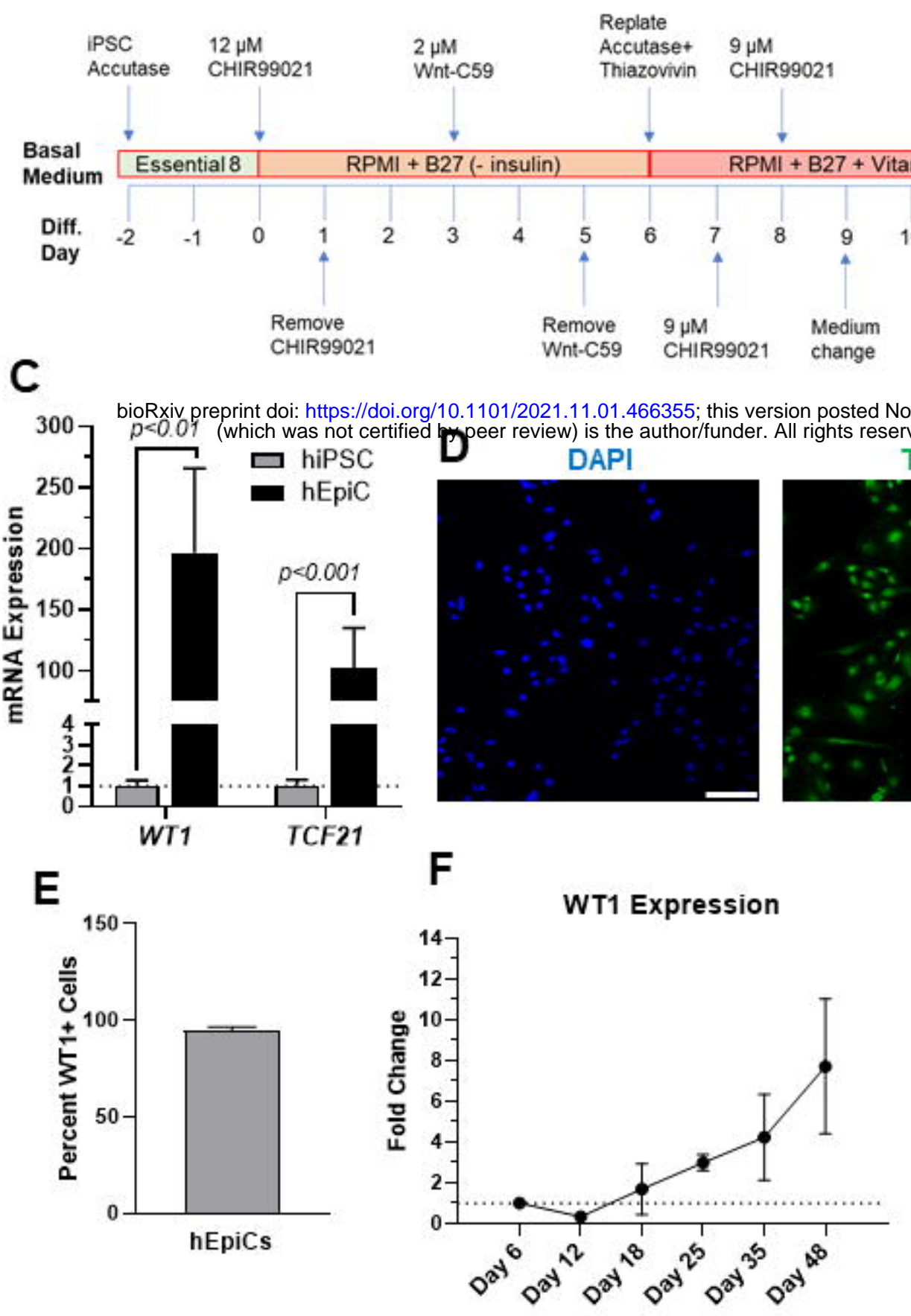

Human iPSC-Derived Epicardial Cell (hEpiC) Differentiation

Replate
Accutase+

Thiazovivin

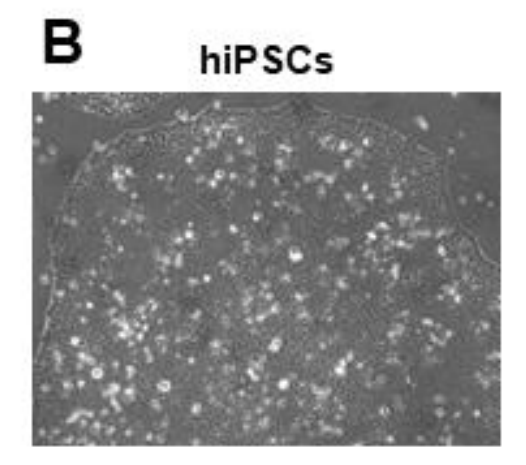

hEpiCs P.2

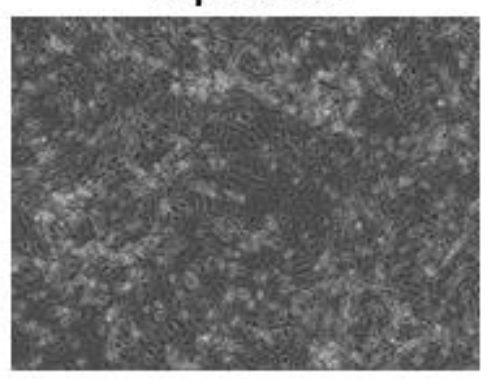

hEpiCs P.3

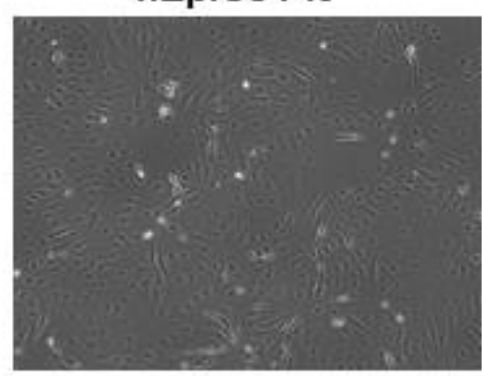

hEpiCs P.4
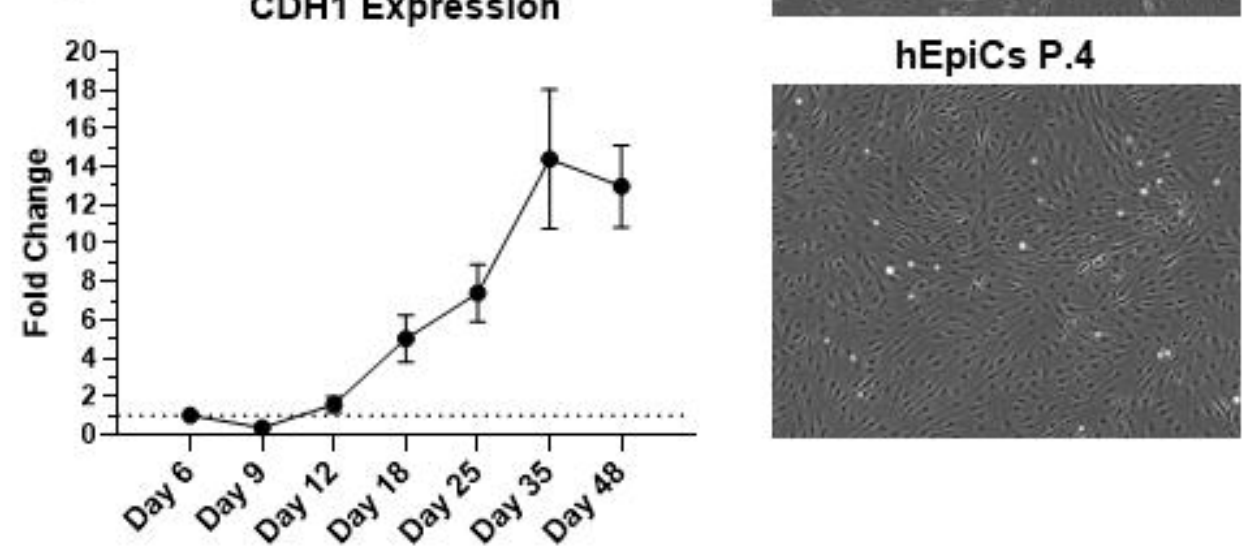

TCF21

NT5E

CDH1

H

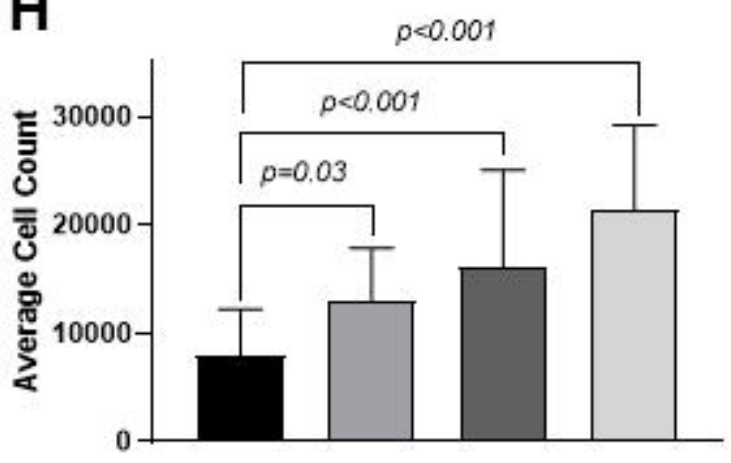

Vitamin C - $+\quad-\quad+$

SB431542 - $\quad-\quad+\quad+$

I

WT1

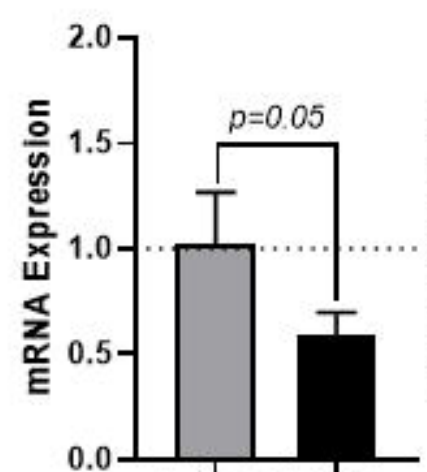

(+) SB (-) SB

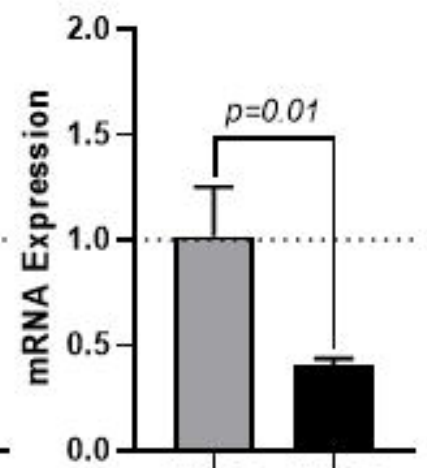

(+) SB (-) SB

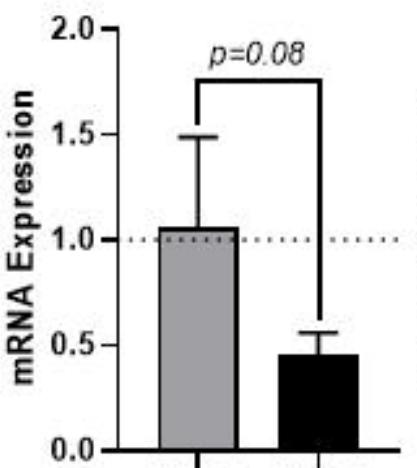

(+) SB (-) SB

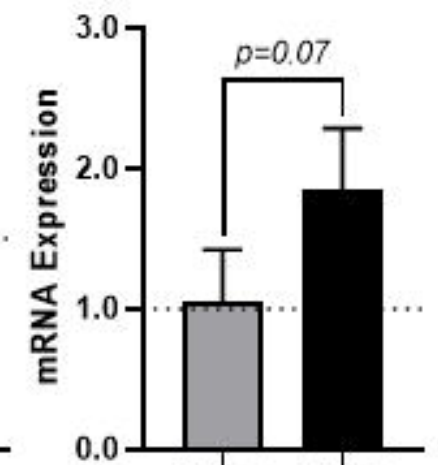

(+) SB (-) SB 
A

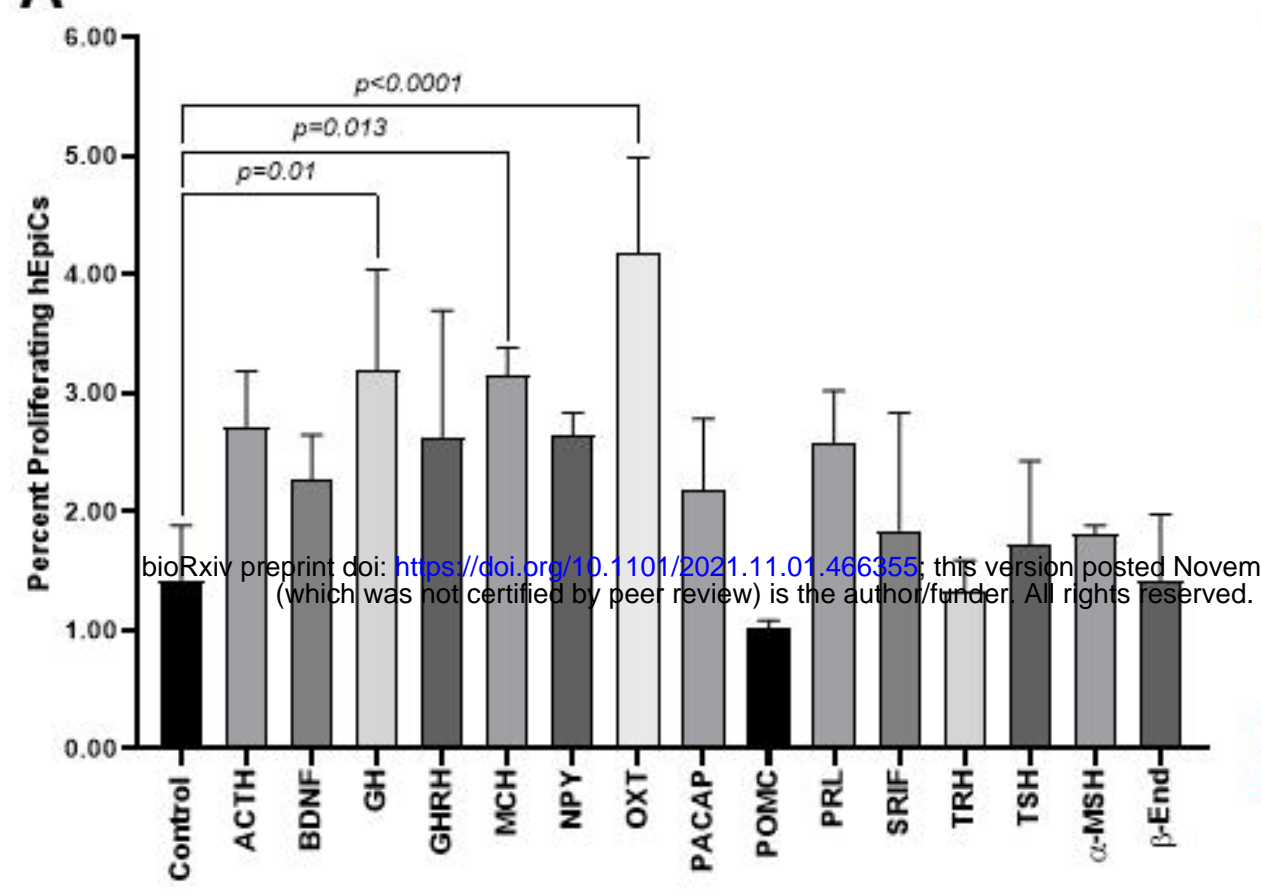

C
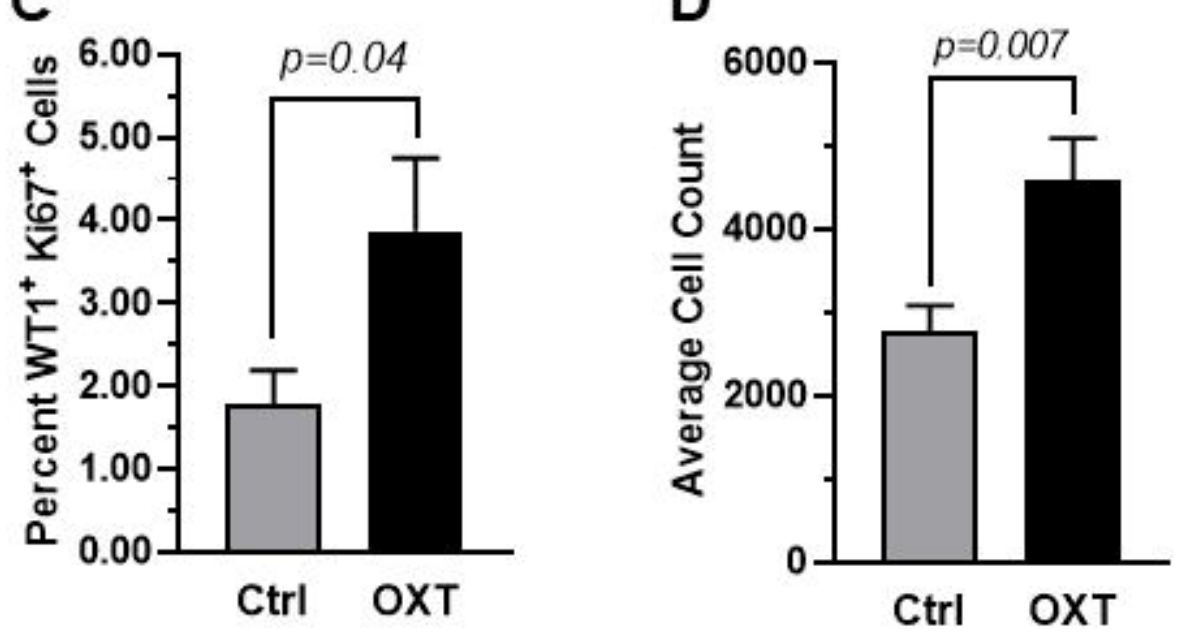

B
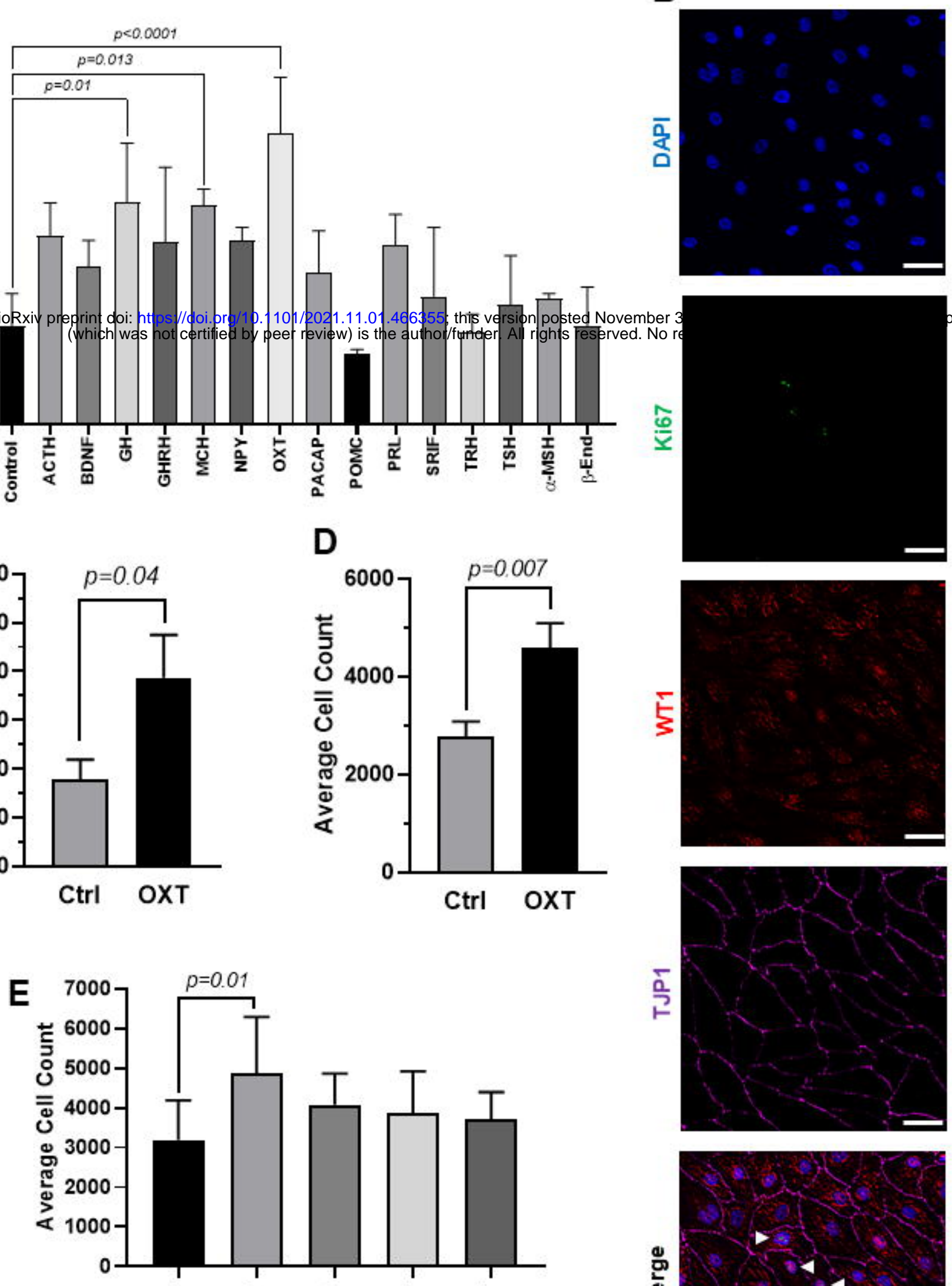

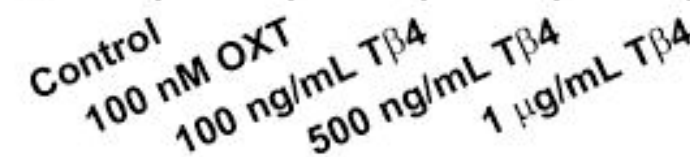
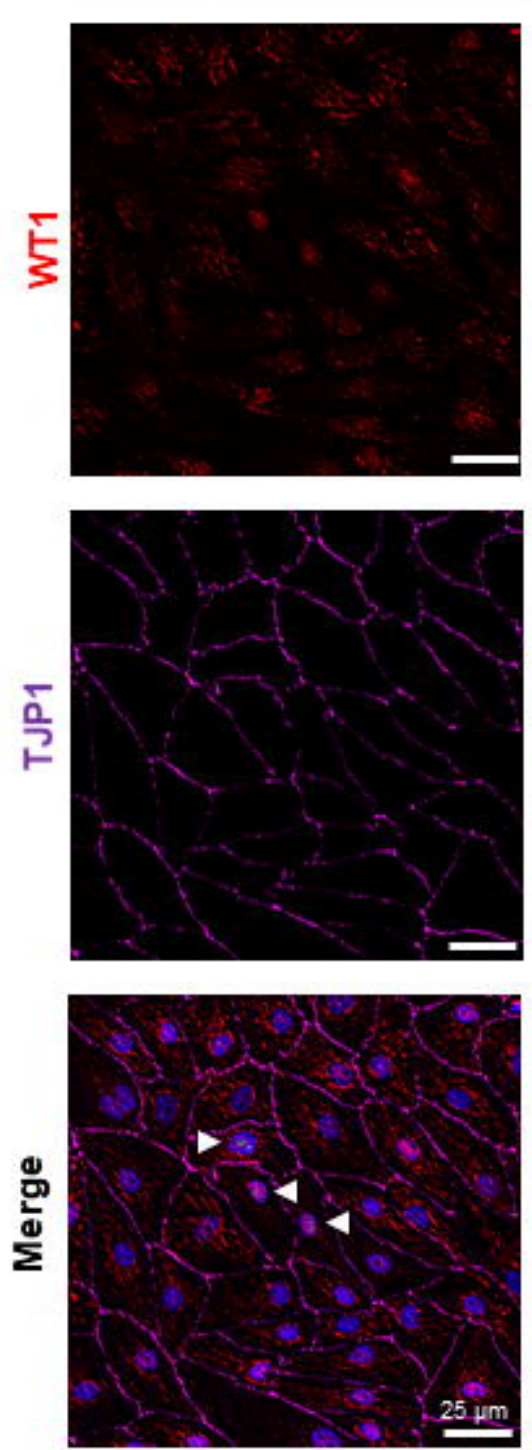

SNAI1

NT5E
Oxytocin
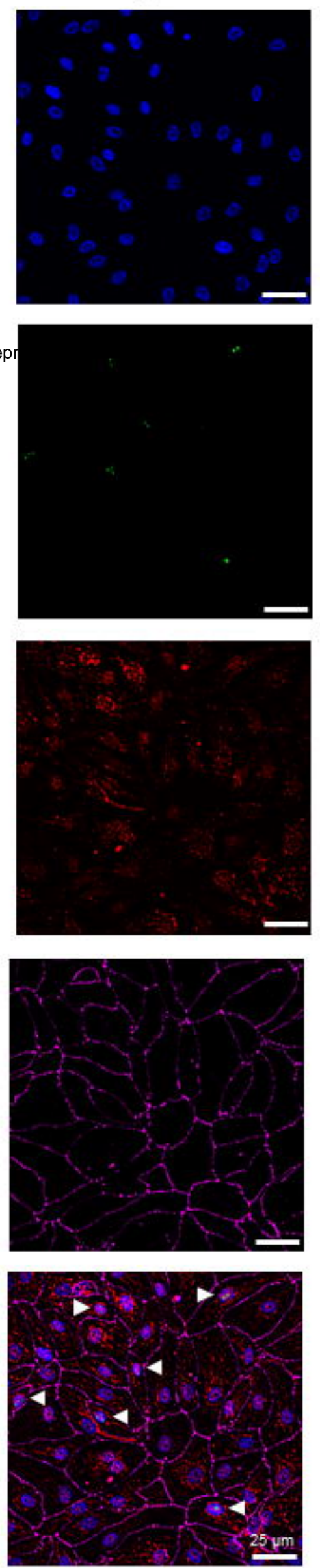

$\mathrm{CDH} 1$

F

TCF21
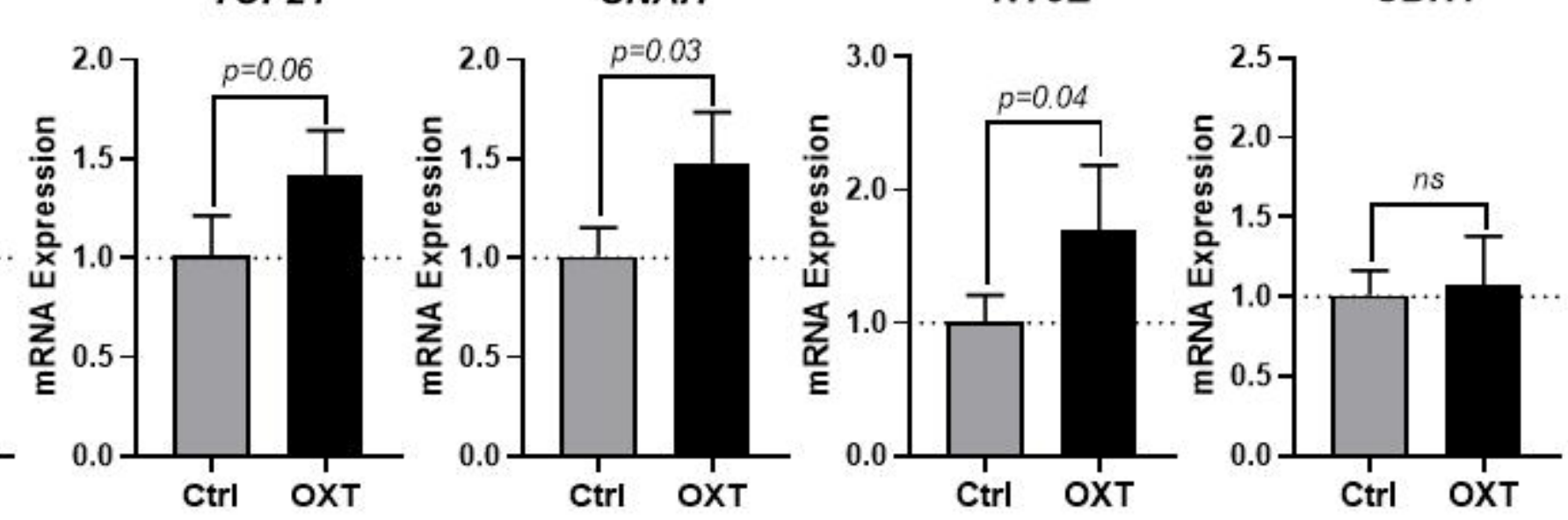


\section{Figure 3}

A

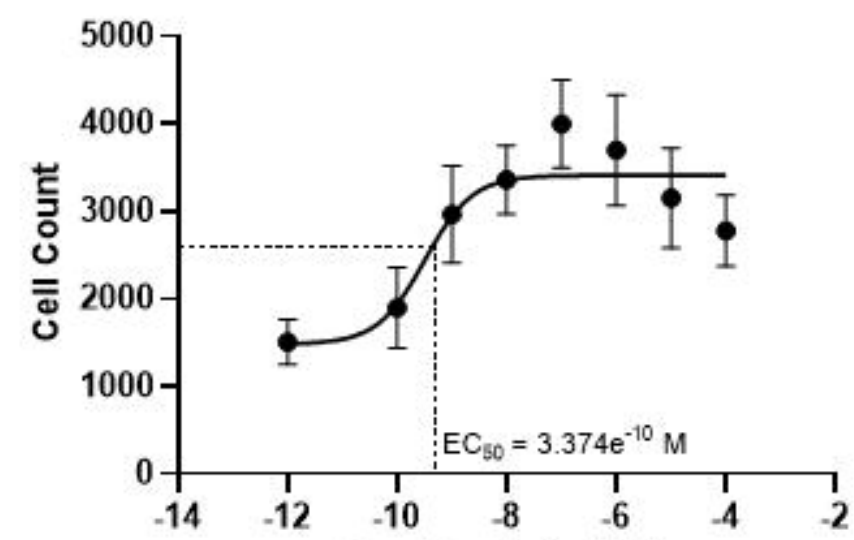

B

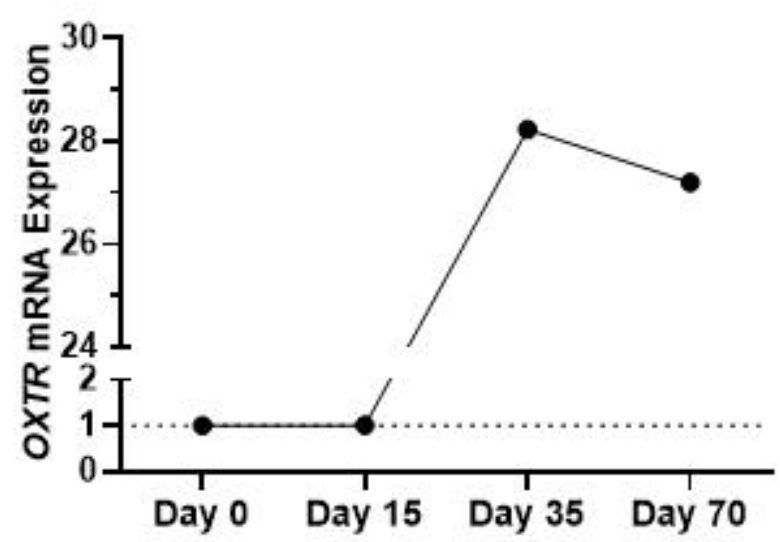

C

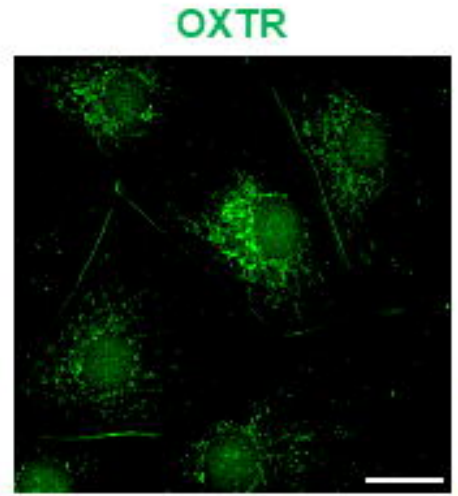

Merge

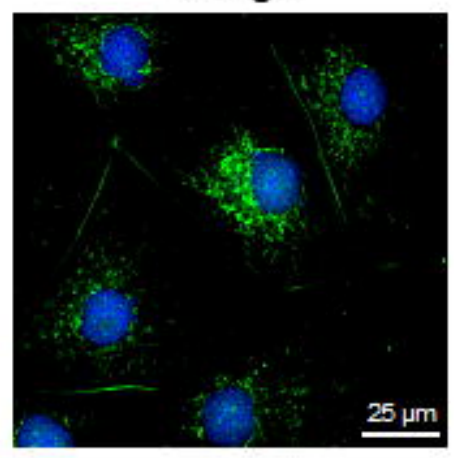

D

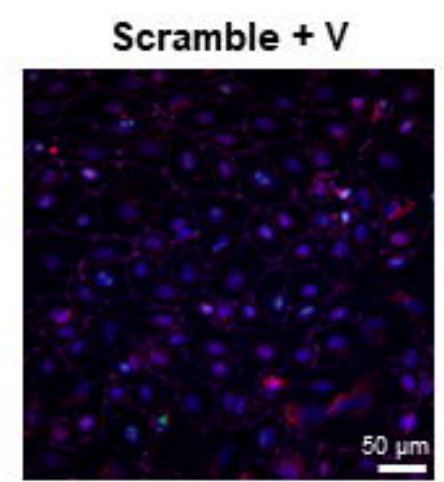

shOXTR + V

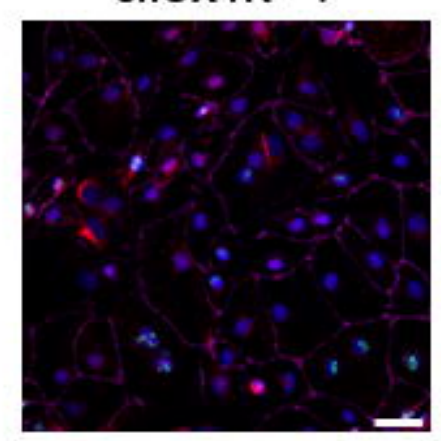

$\mathbf{F}$

WT1
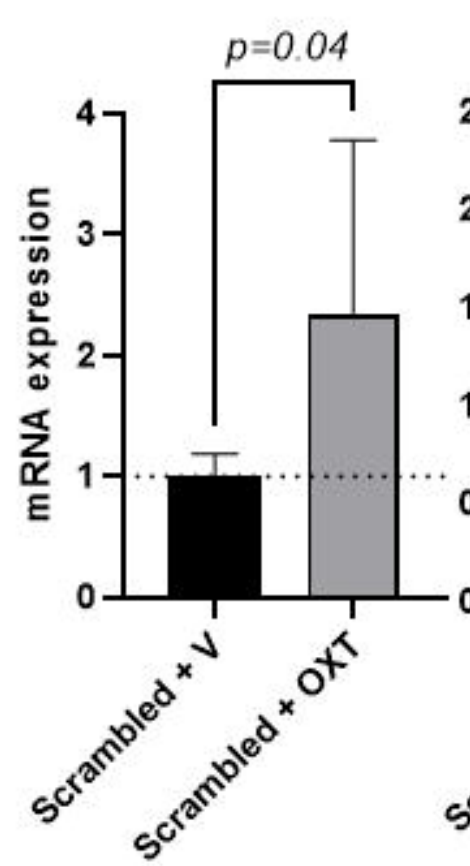

TCF21 SNAI1

Scramble + OXT

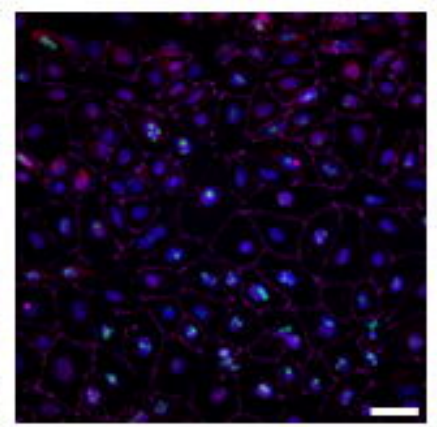

ShOXTR + OXT
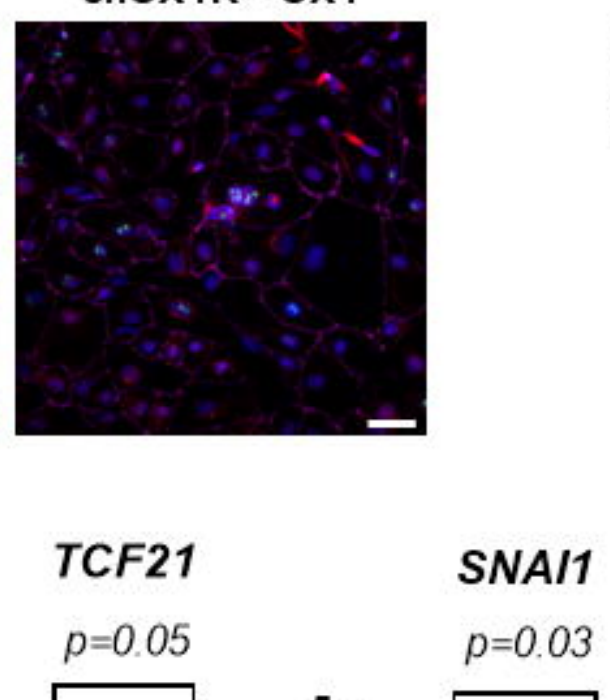

E

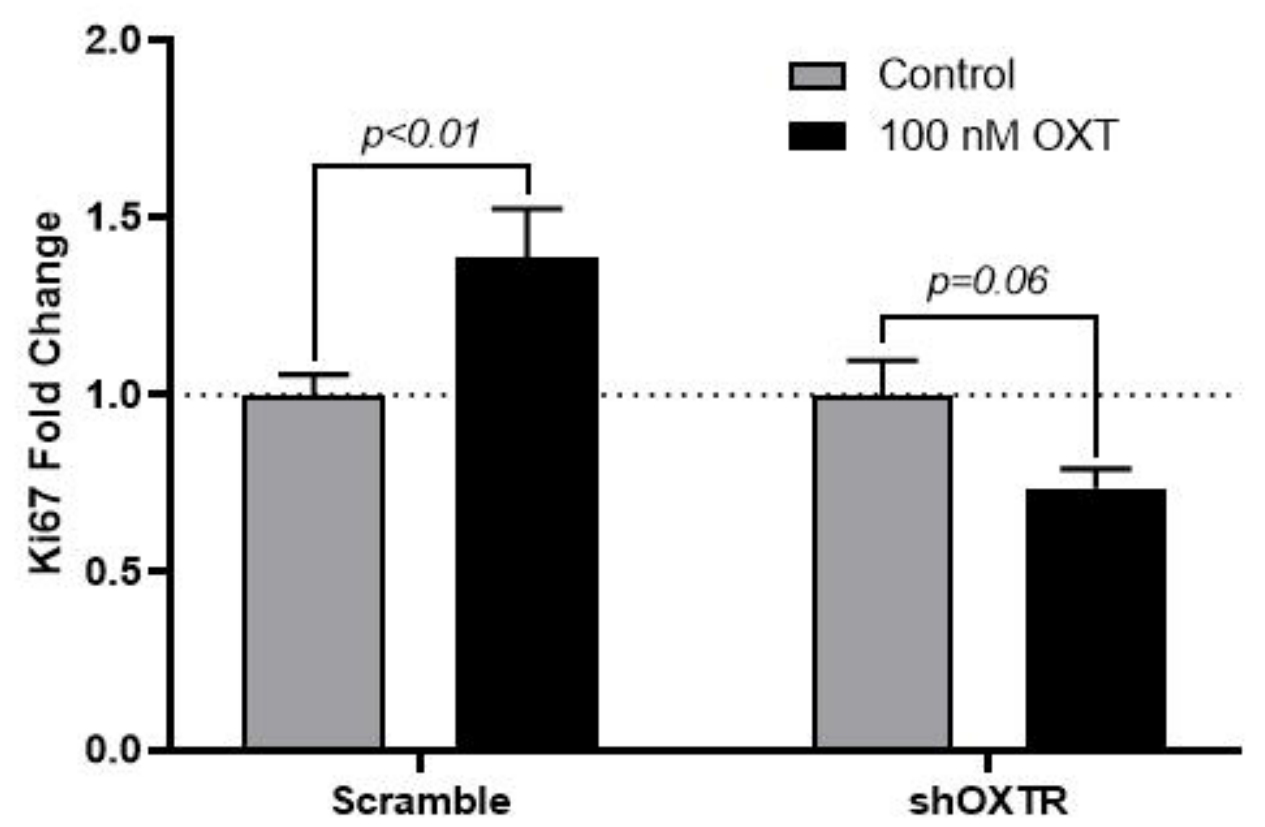

G

Cell Line

TCF21
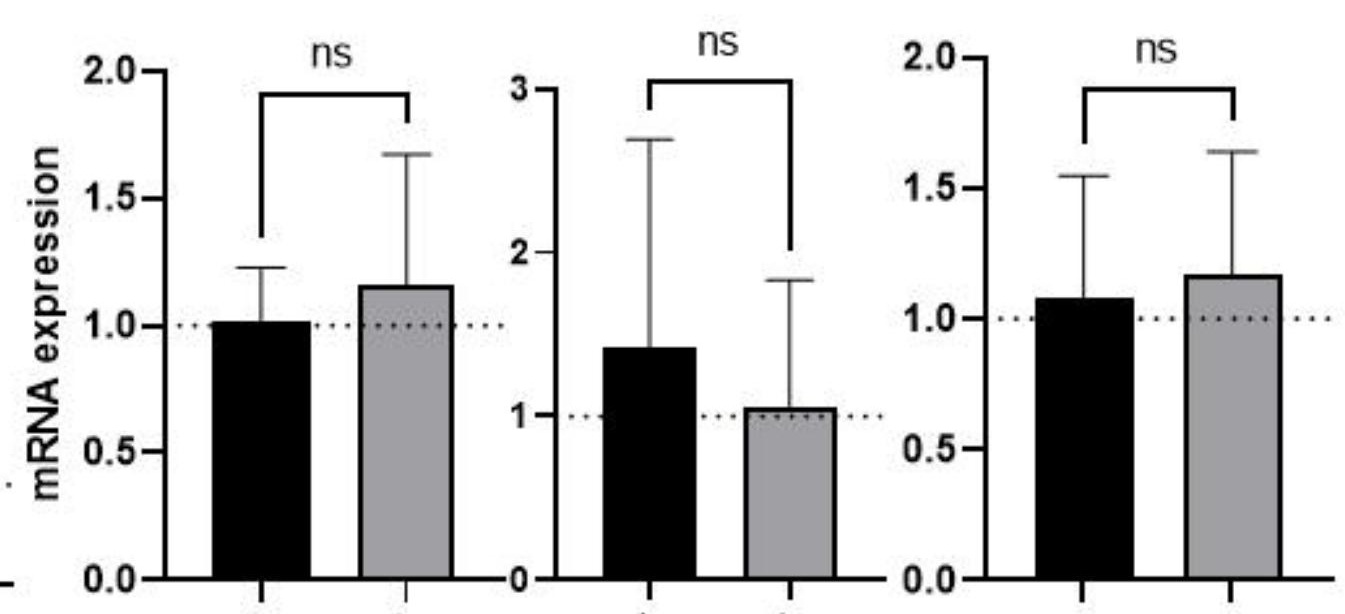
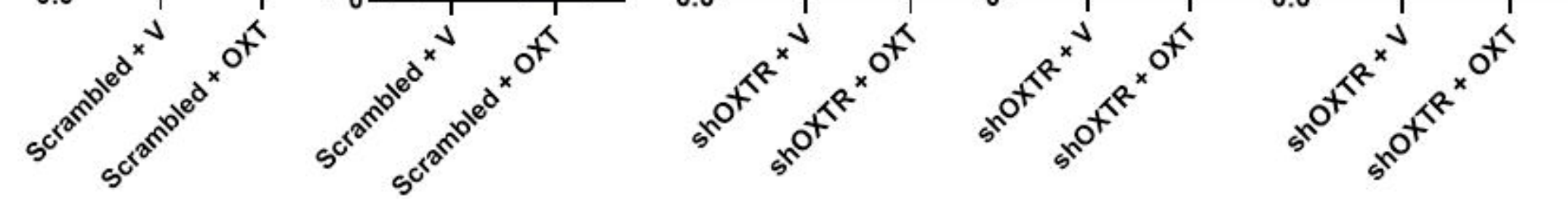


\section{Figure 4}

A

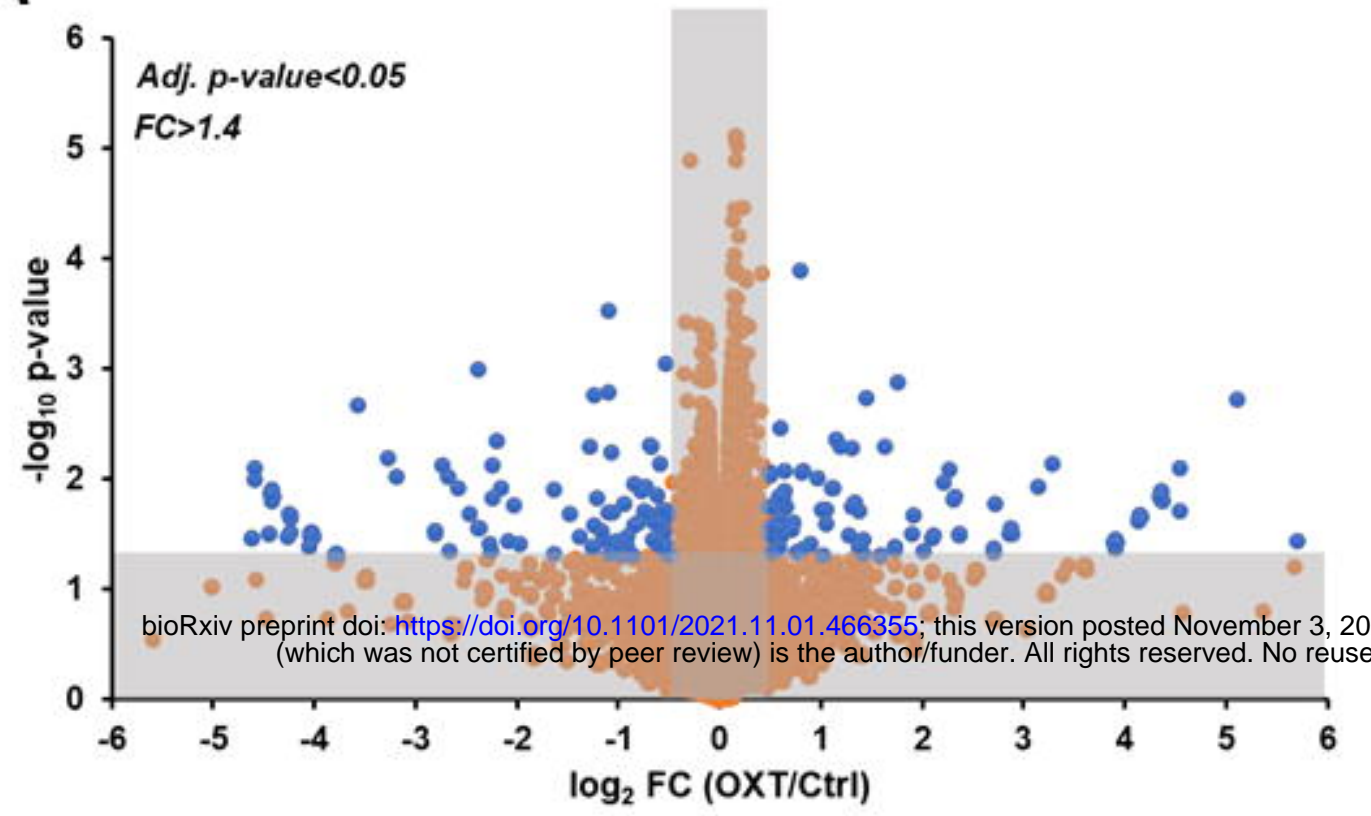

C
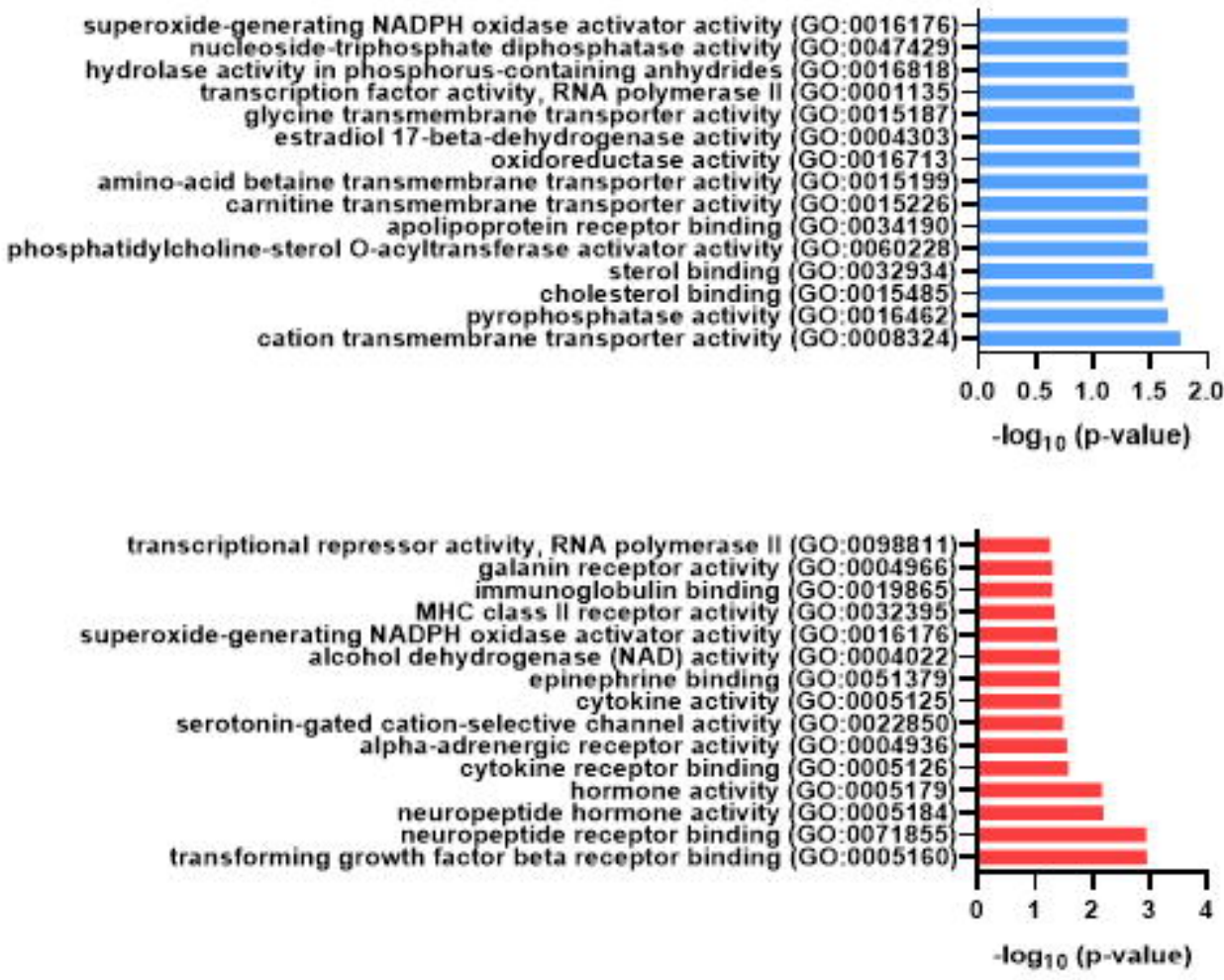

B

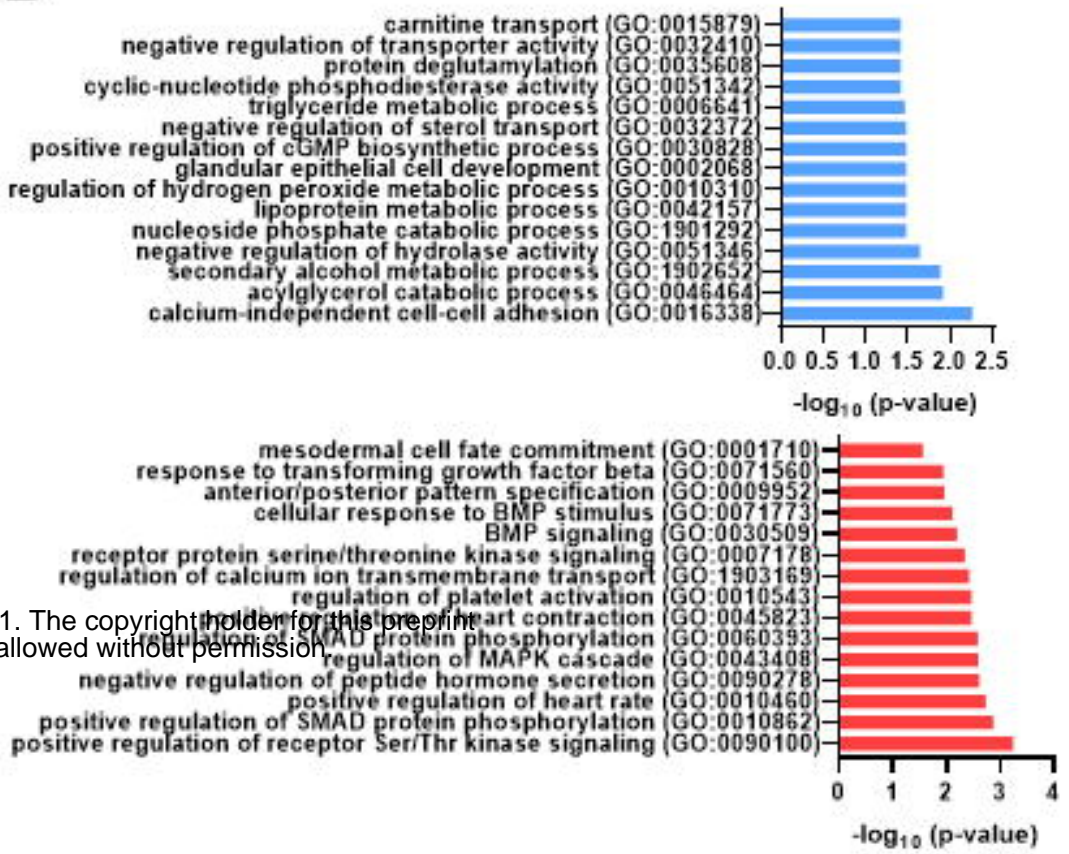

D

LEFTY2

GDF15

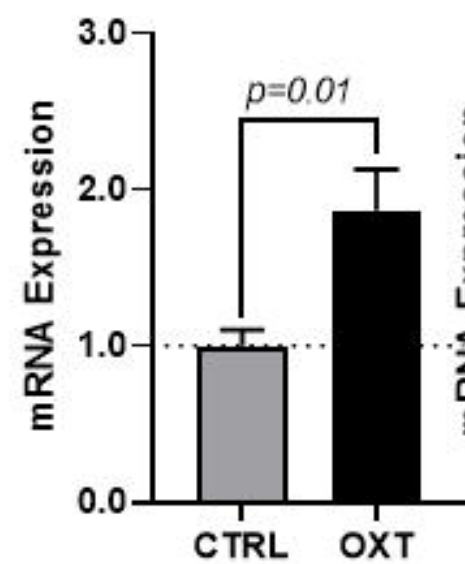

INHBB
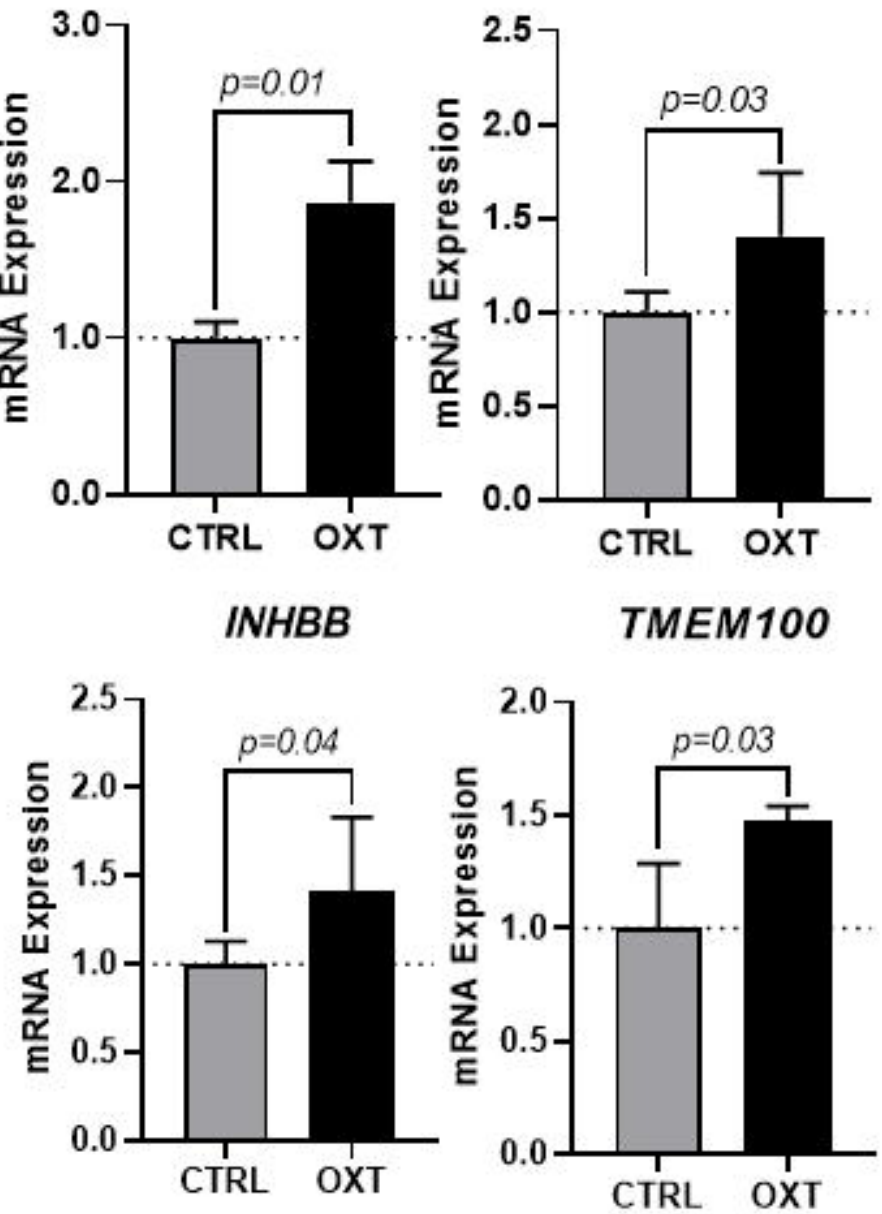
A myl7-DsRed2
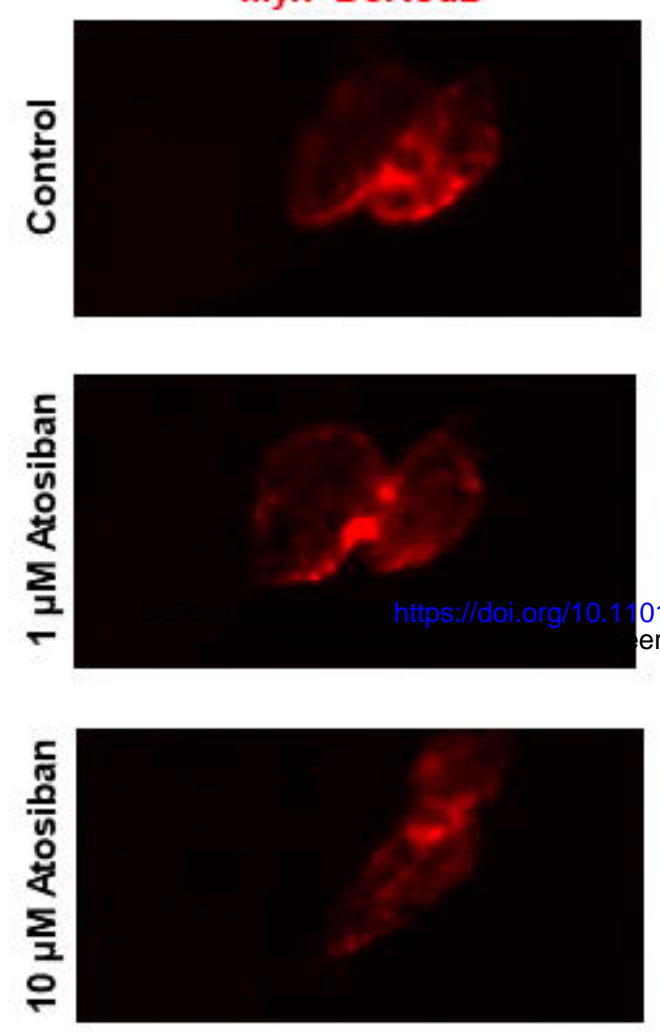

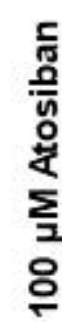

tcf21-nls-eGFP
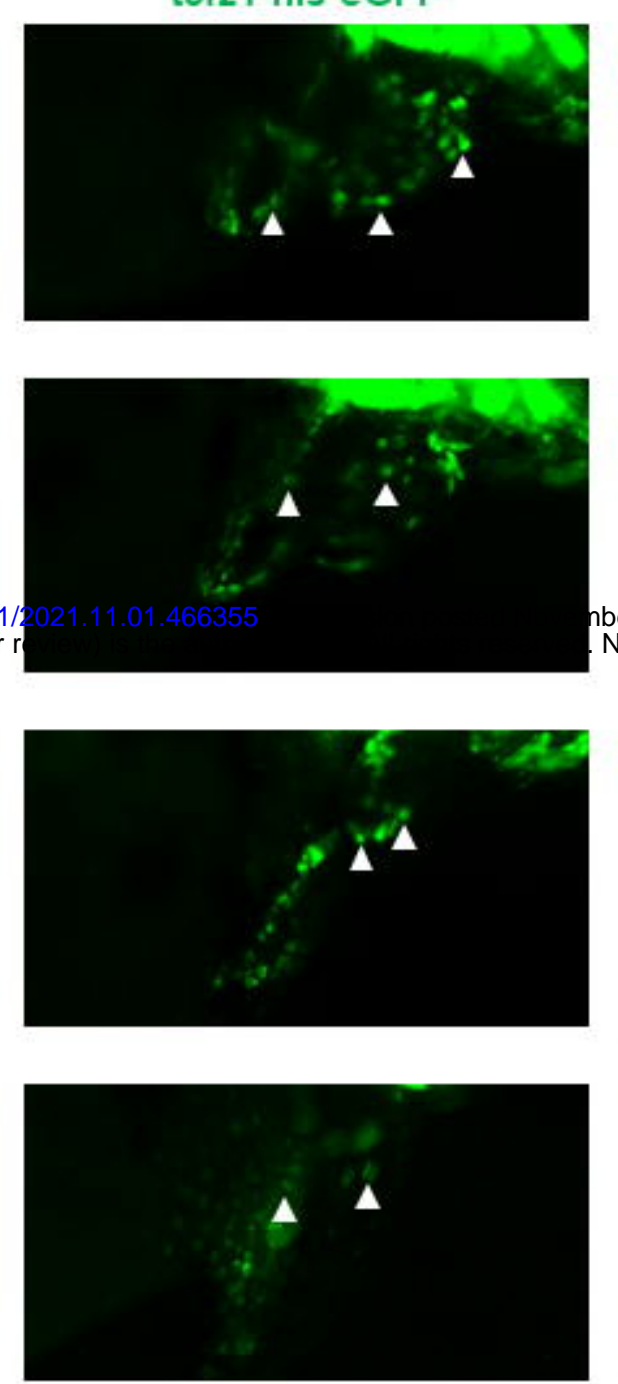

Merge
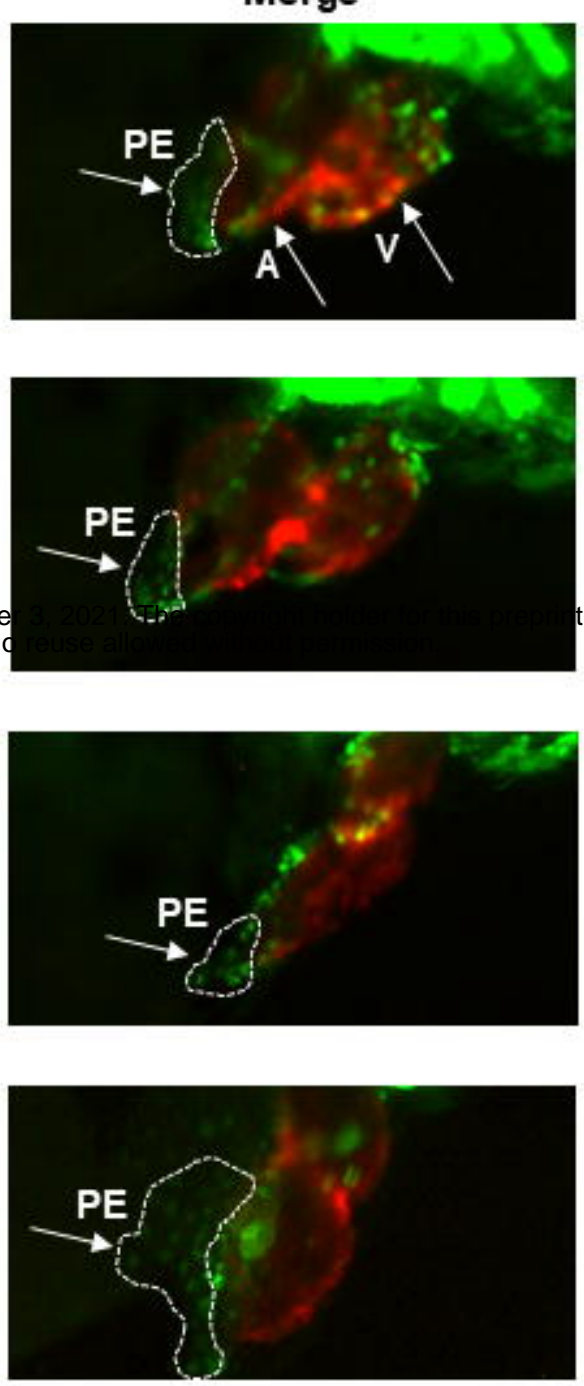

B

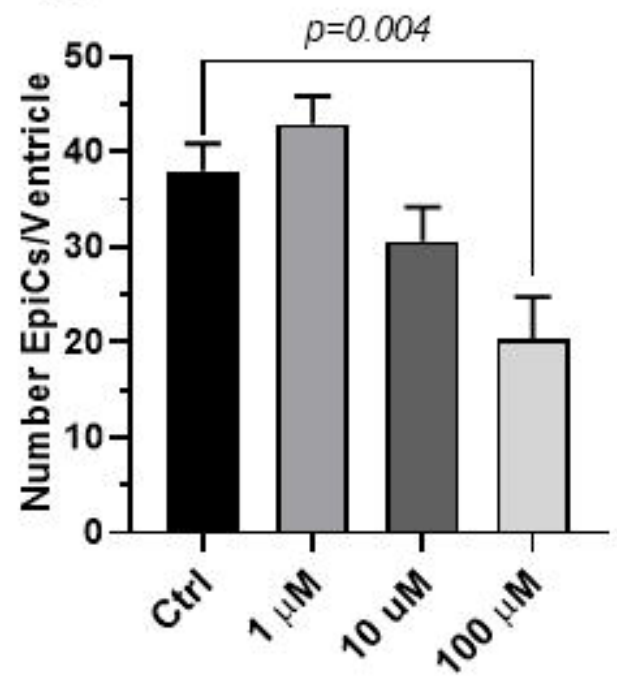

C

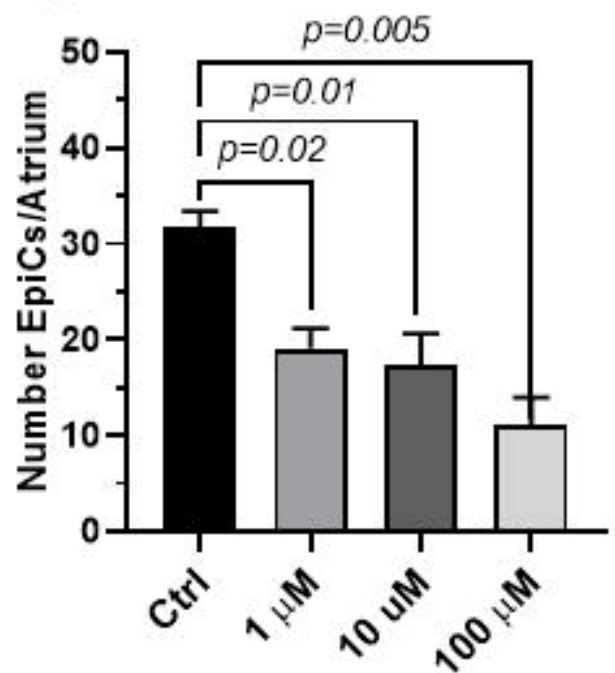




\section{Figure 6}

A

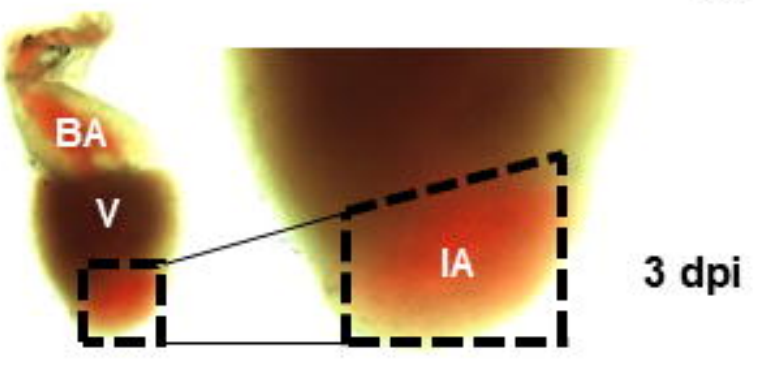

C

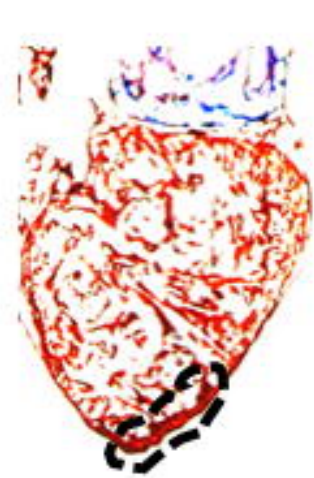

Cryoinjury $+\mathrm{H} 2 \mathrm{O}$

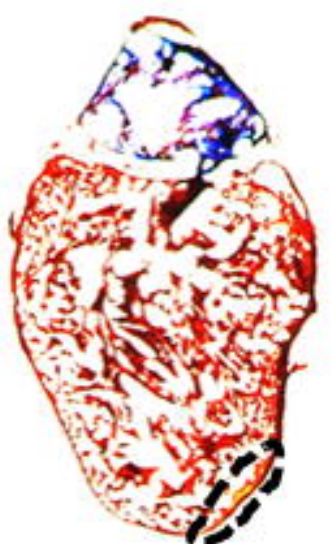

Cryoinjury + L-368,899
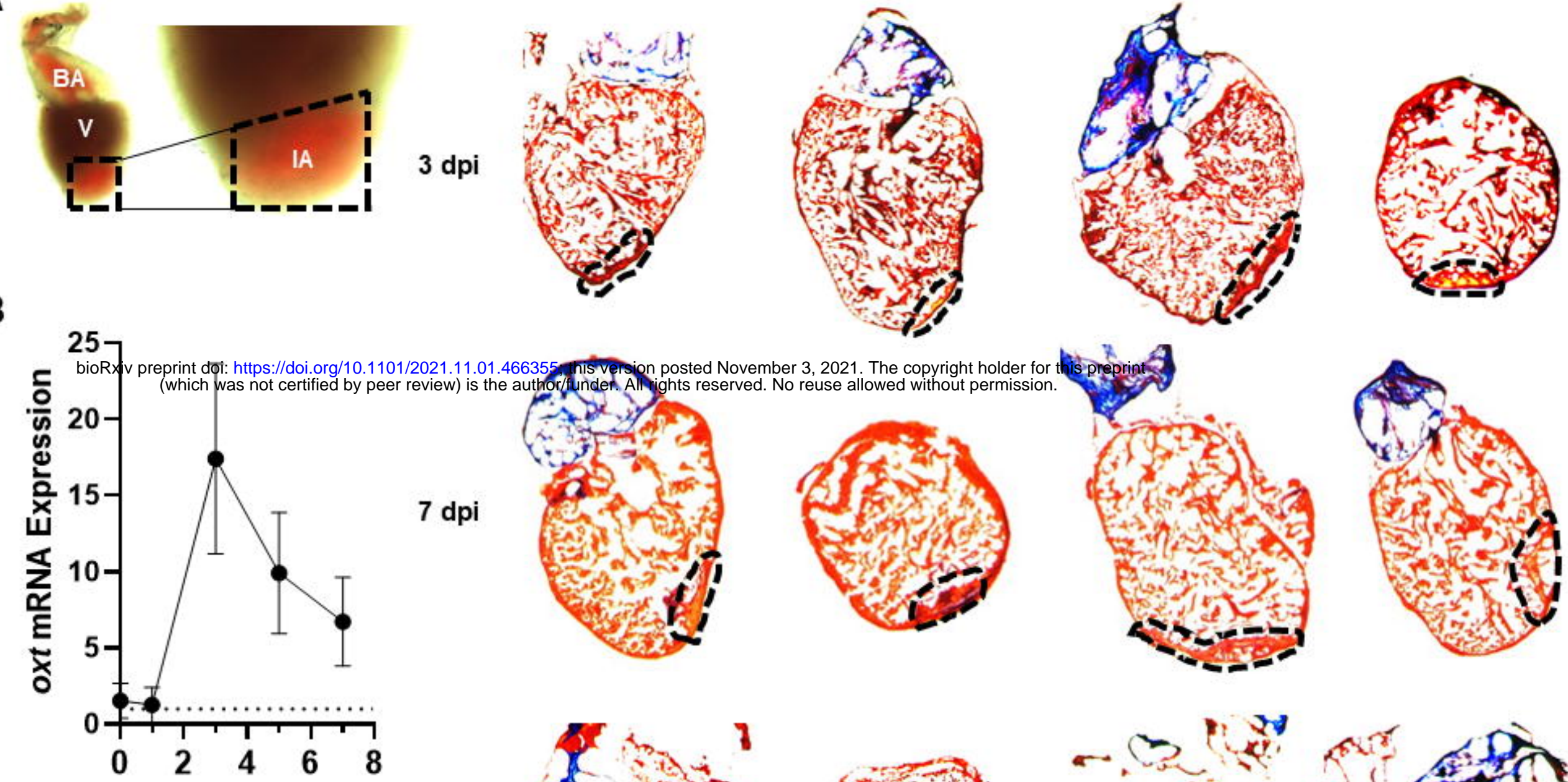

Days Post-Injury

7 dpi
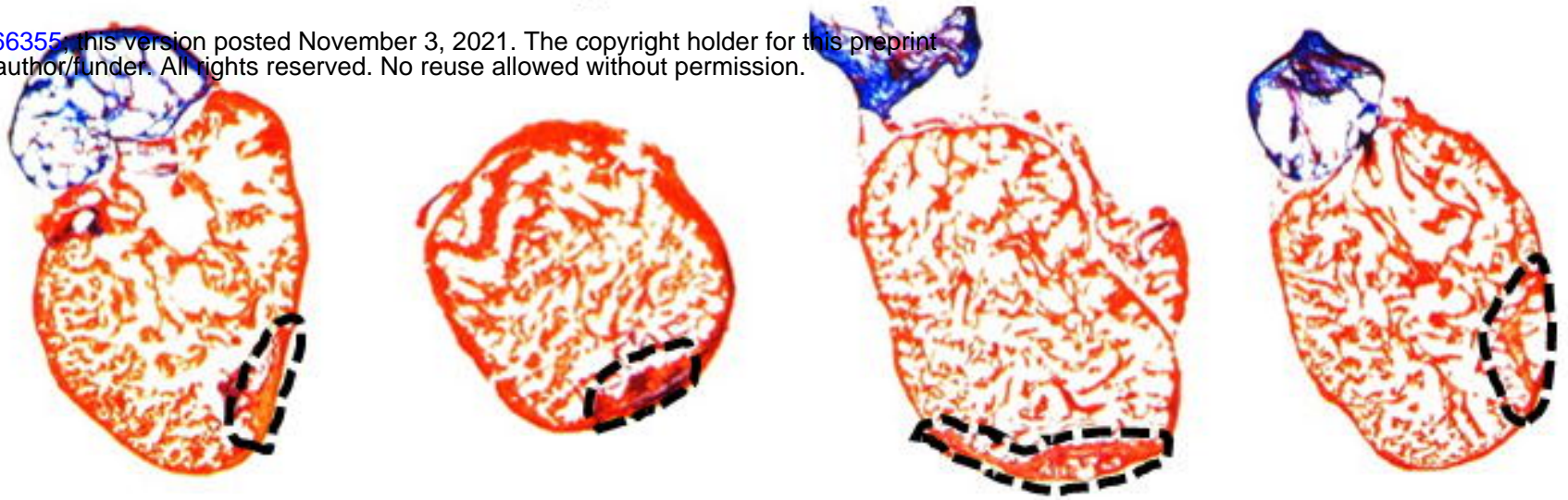

$14 \mathrm{dpi}$
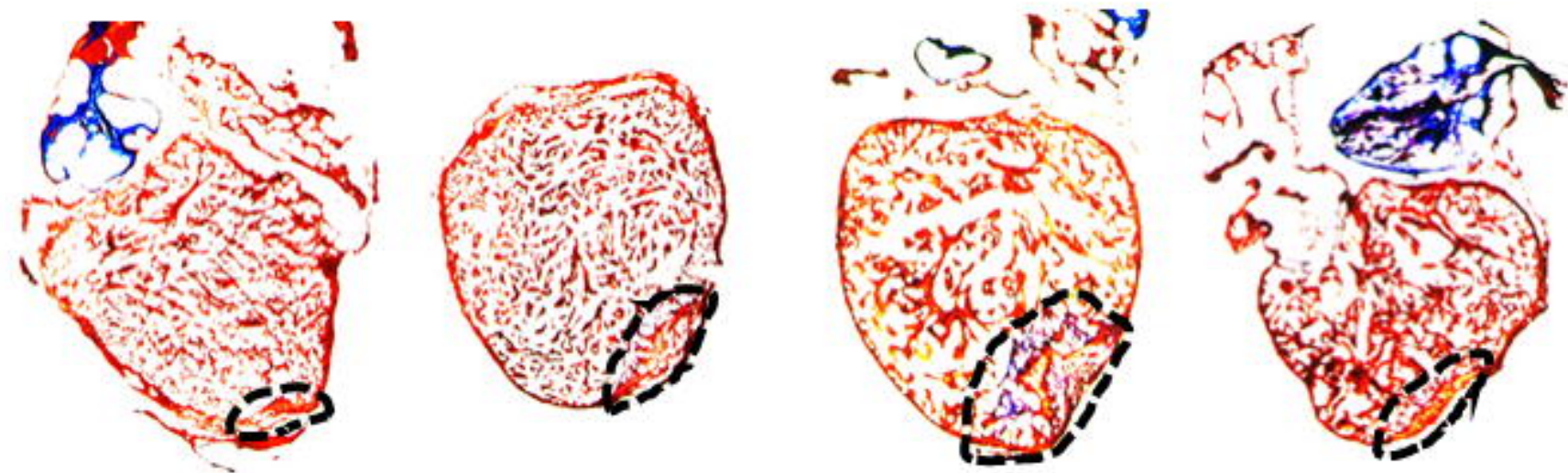

D

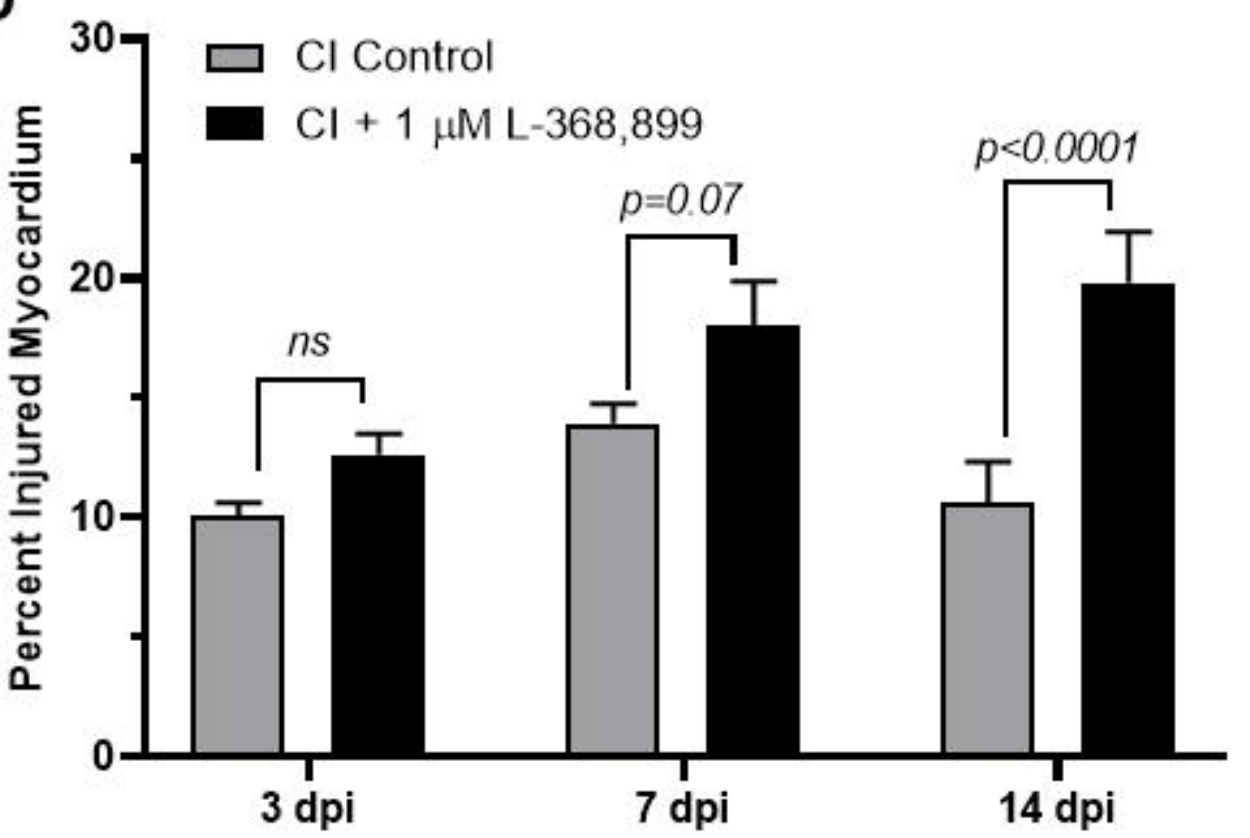

E
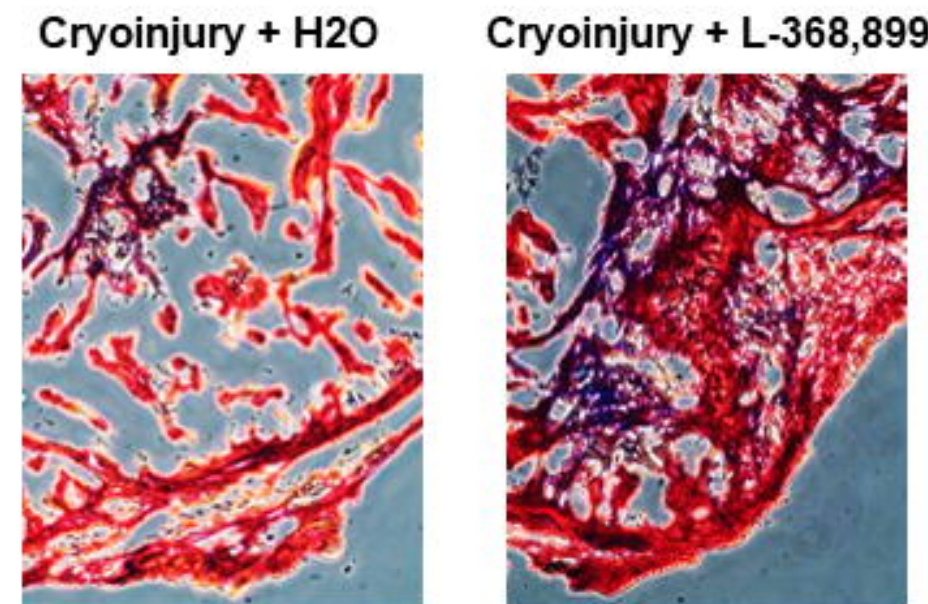
A

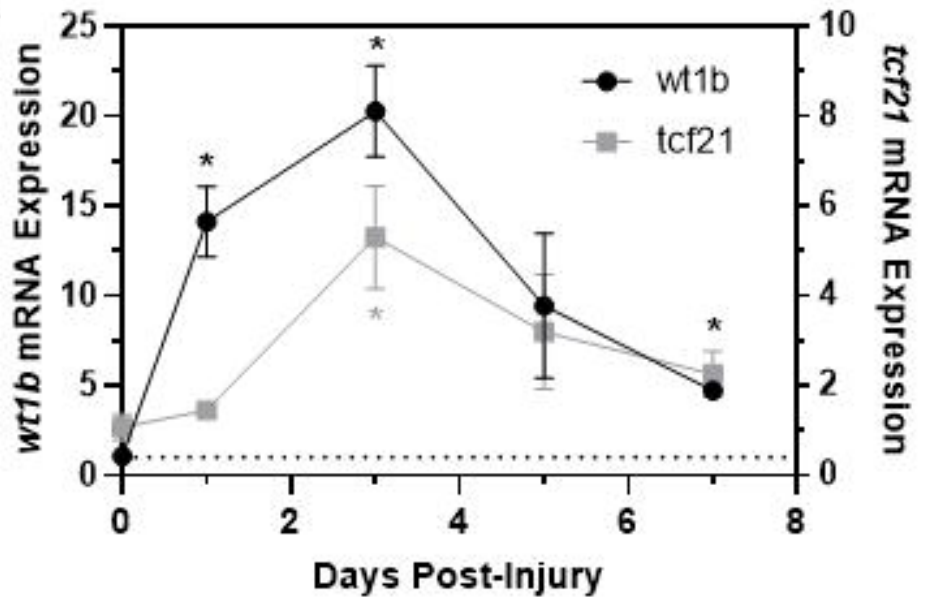

C

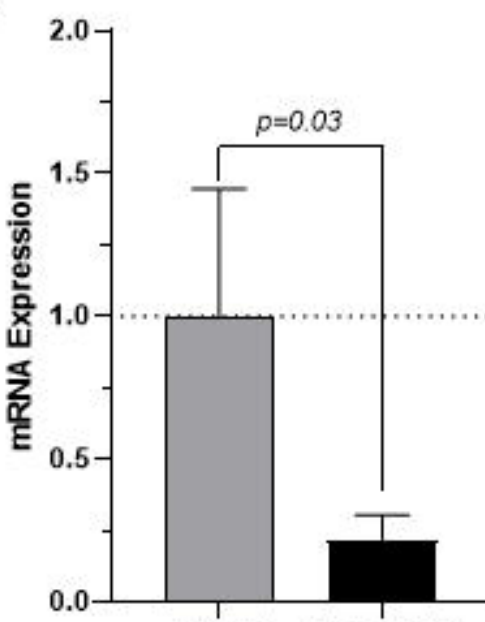

tcf 21

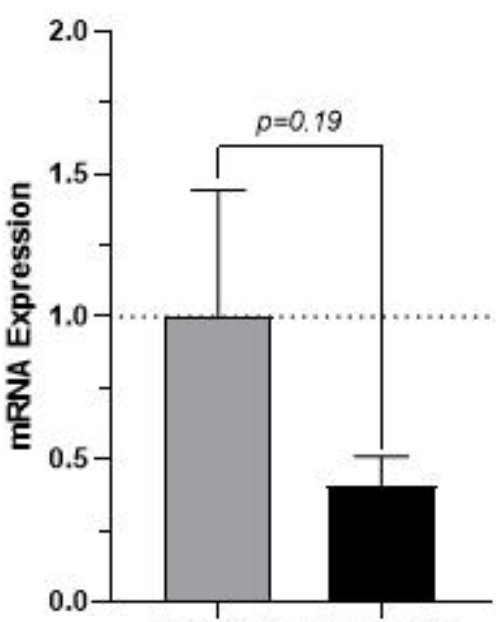

0x Merge
TNNT2
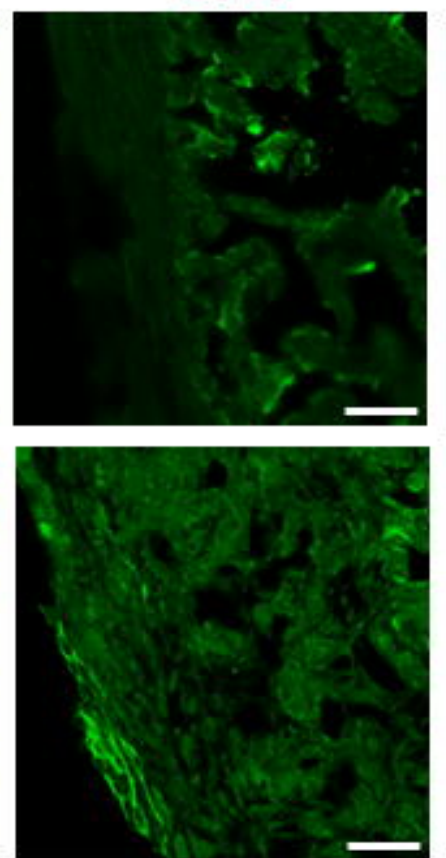

H3P
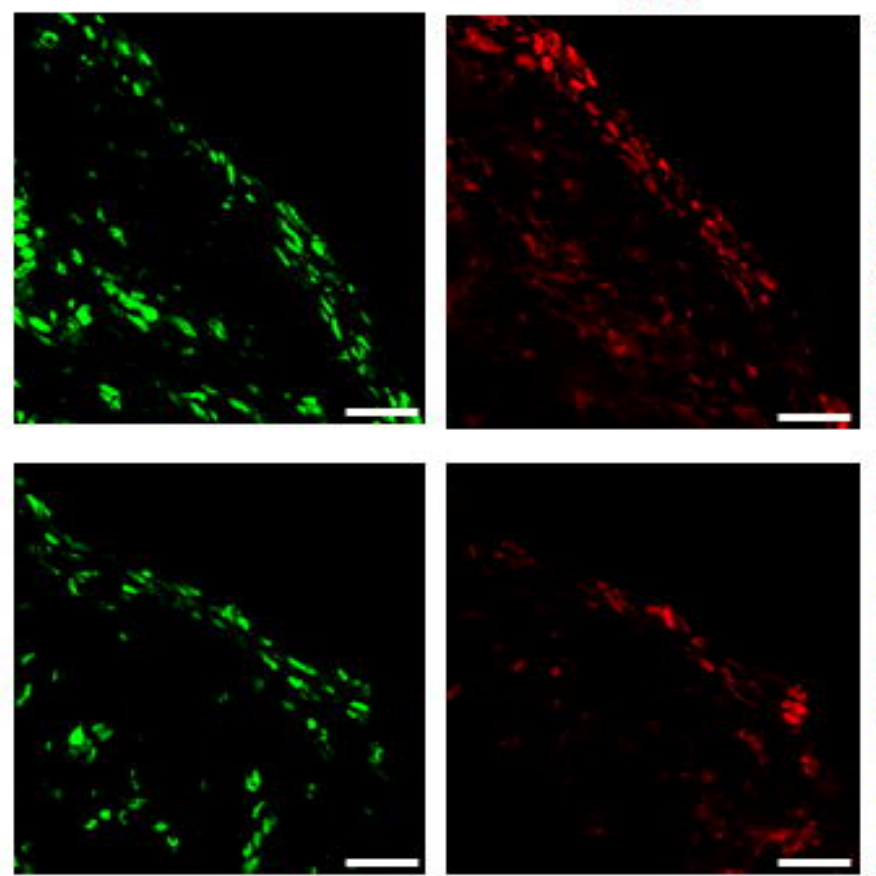

snai1a

F
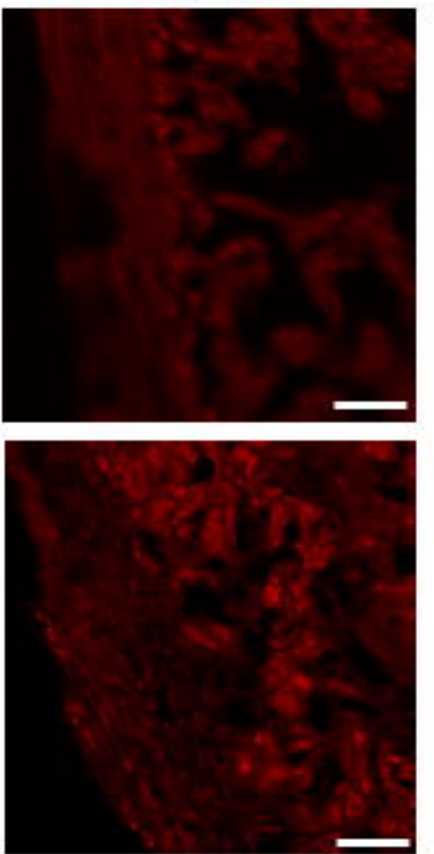

WT1

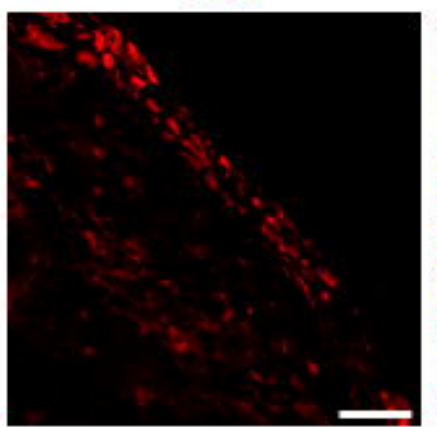

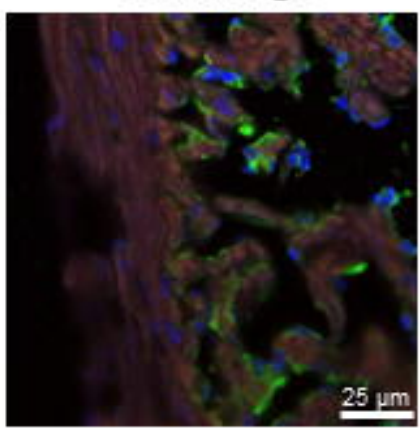

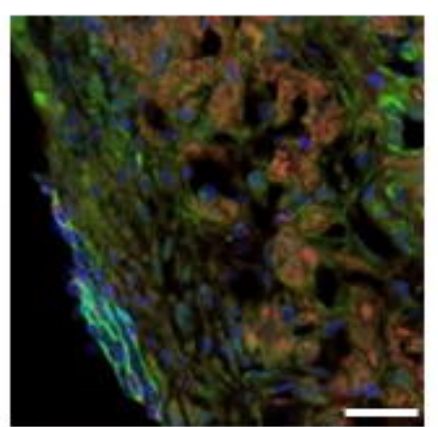

60x Merge
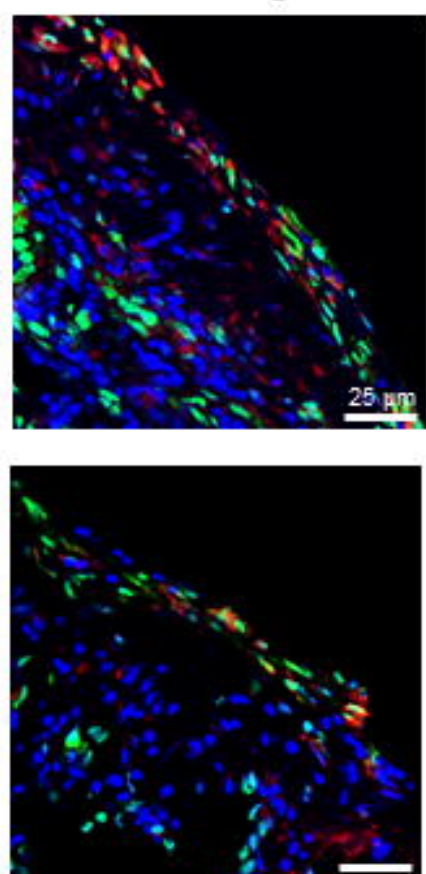

snai2

E

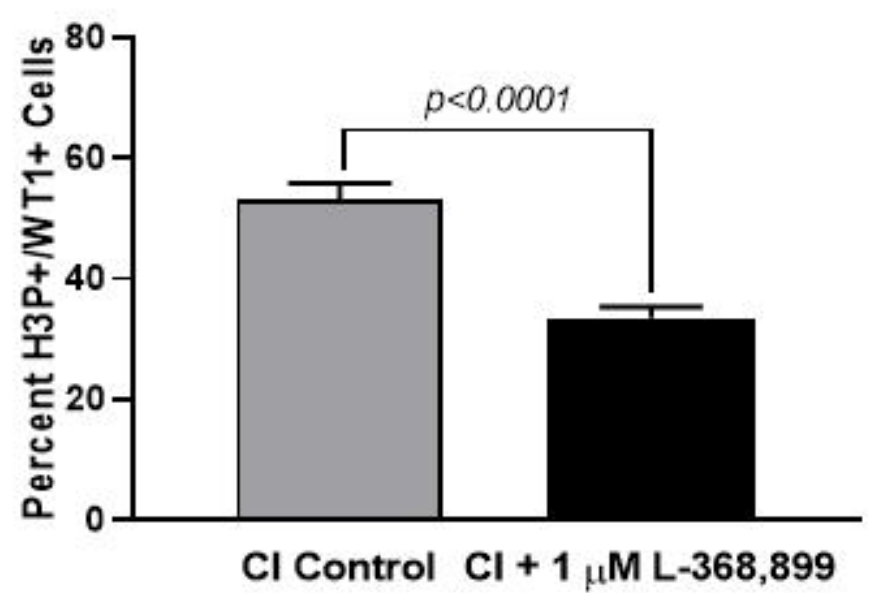

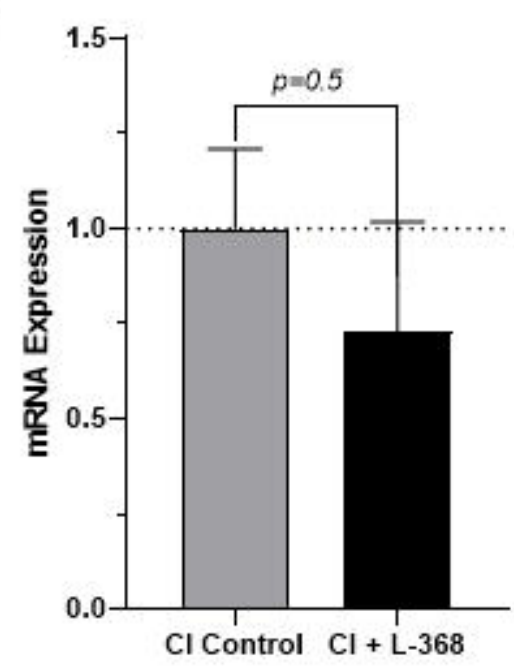

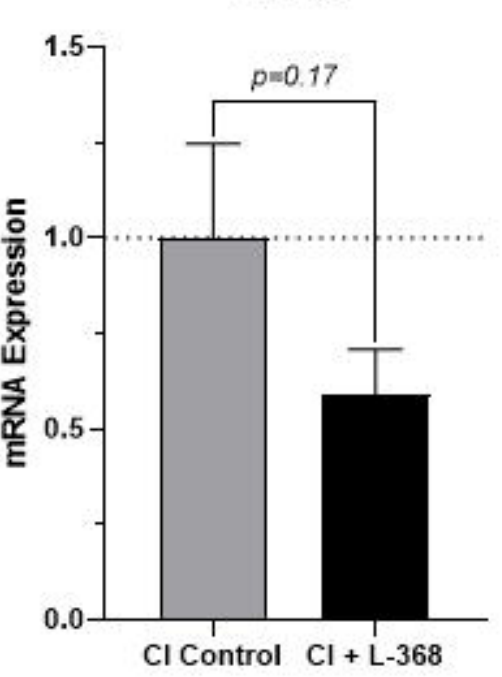

